\title{
QUASI-CONFORMAL RIGIDITY OF MULTICRITICAL MAPS
}

\author{
WENJUAN PENG AND LEI TAN
}

\begin{abstract}
We combine the enhanced nest constructed by Kozlovski, Shen and van Strien, and the analytic method proposed by Avila, Kahn, Lyubich and Shen to prove quasi-conformal rigidity properties of multicritical maps.
\end{abstract}

\section{INTRODUCTION}

Rigidity is one of the fundamental and remarkable phenomena in holomorphic dynamics. The general rigidity problem can be posed as

Rigidity problem ([20]). Any two combinatorially equivalent rational maps are quasi-conformally equivalent. Except for the Lattès examples, the quasi-conformal deformations come from the dynamics of the Fatou set.

In the quadratic polynomial case, the rigidity problem is equivalent to the MLC conjecture, asserting that the Mandelbrot set is locally connected. This conjecture is stronger than the hyperbolic conjecture (cf. 6]). In 1990, Yoccoz 9] proved MLC for the finitely renormalizable parameter values. MLC is also known to be true at many infinitely renormalizable parameters (see works of Inou-Shishikura, Kahn-Lyubich and Levin, [10 13, 18, 20], among others).

For higher degree polynomials, Kozlovski, Shen and van Strien gave a proof in [16] of the rigidity for real polynomials with all critical points real. In 2, Avila, Kahn, Lyubich and Shen proved that any unicritical polynomial $f_{c}: z \mapsto z^{d}+c$ which is at most finitely renormalizable and has only repelling periodic points is combinatorially rigid, which implies that the connectedness locus (the Multibrot set) is locally connected at the corresponding parameter values. Kozlovski and van Strien proved that topologically conjugate nonrenormalizable polynomials are quasi-conformally conjugate (cf. [17]).

The rigidity problem for rational maps with Cantor Julia sets is totally solved (cf. 32, 33]). In 33, Zhai took advantage of a length-area method introduced by Kozlovski, Shen and van Strien (cf. [16]) to prove the quasi-conformal rigidity for rational maps with Cantor Julia sets.

In this article, we shall investigate proper complex box mappings or in short proper box mappings defined below. We have first studied the rigidity problem of such mappings with a unique critical point (see [24]). The current work deals with the general case.

Received by the editors January 20, 2012 and, in revised form, January 30, 2013.

2010 Mathematics Subject Classification. Primary 37F10, 37F20.

Key words and phrases. Holomorphic dynamics, quasi-conformal rigidity, multicritical maps, enhanced nest.

The first author was supported by the NSF of China under grants No. 11101402 and No. 11231009, by the PSSF of China under grant No. 201003020 and by SRF for ROCS, SEM.

The second author was supported by Geanpyl Pays de la Loire and ANR LAMBDA. 
A proper box mapping is a map $f: \mathbf{U} \rightarrow \mathbf{V}$ such that:

$\mathbf{V}$ is the union of finitely many quasi-disks

in the complex plane $\mathbb{C}$ with pairwise disjoint closures,

$\mathbf{U}$ is compactly contained in $\mathbf{V}$,

and is the union of finitely many quasi-disks with pairwise disjoint closures;

$f: \mathbf{U} \rightarrow \mathbf{V}$ is a proper holomorphic map with all critical points

contained in $\mathbf{K}_{f}:=\left\{z \in \mathbf{U} \mid f^{n}(z) \in \mathbf{U} \forall n\right\}$, such that

each connected component of $\mathbf{V}$

contains at most one connected component of $\mathbf{K}_{f}$ having critical points.

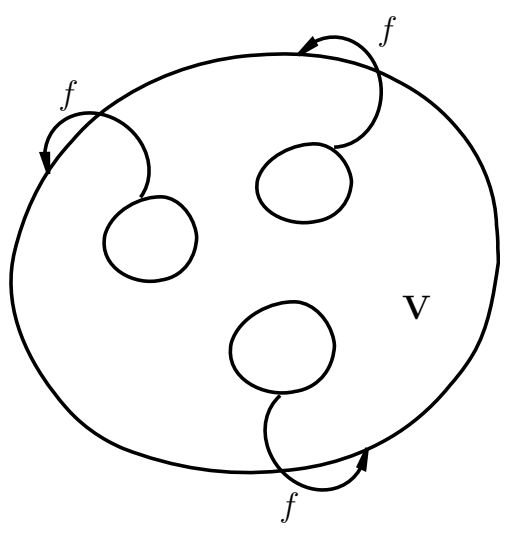

FiguRE 1. A proper box mapping with $\mathbf{V}$ a single quasi-disk.

A proper box mapping is a particular case of a complex box mappings in the sense of [16], where a component 1 of $\mathbf{U}$ is allowed to be either equal to a component of $\mathbf{V}$ or properly contained in $\mathbf{V}$.

Proper box mappings appear naturally as induced dynamical systems, of a rational map having a completely invariant (super)attracting Fatou component. See Appendix A for a concrete example.

Definition. Let $f: \mathbf{U} \rightarrow \mathbf{V}$ and $\tilde{f}: \tilde{\mathbf{U}} \rightarrow \tilde{\mathbf{V}}$ be two proper box mappings.

- We say that $f$ and $\tilde{f}$ are quasi-conformally conjugate off $\mathbf{K}_{f}$ if there is a (partial) qc (qc is an abbreviation of quasi-conformal) homeomorphism $H: \mathbf{V} \backslash \mathbf{K}_{f} \stackrel{\approx}{\longrightarrow} \tilde{\mathbf{V}} \backslash \mathbf{K}_{\tilde{f}}$ so that $H \circ f=\tilde{f} \circ H$ on $\mathbf{U} \backslash \mathbf{K}_{f}$, i.e.,

$$
\begin{array}{llll}
\mathbf{U} \backslash \mathbf{K}_{f} & \stackrel{H}{\longrightarrow} & \tilde{\mathbf{U}} \backslash \mathbf{K}_{\tilde{f}} & \\
f \downarrow & & \downarrow \tilde{f} & \text { commutes. } \\
\mathbf{V} \backslash \mathbf{K}_{f} & \rightarrow & \tilde{\mathbf{V}} \backslash \mathbf{K}_{\tilde{f}} &
\end{array}
$$

- Let $\operatorname{int} \mathbf{K}_{f}$ denote the interior of $\mathbf{K}_{f}$. We say that $f$ and $\tilde{f}$ are quasiconformally conjugate off int $\mathbf{K}_{f}$ if there is a (global) qc homeomorphism

\footnotetext{
${ }^{1}$ In this article, for simplicity, by a 'component', we mean a 'connected component'.
} 
$\tilde{H}: \mathbf{V} \stackrel{\approx}{\longrightarrow} \tilde{\mathbf{V}}$ so that $\tilde{H} \circ f=\tilde{f} \circ \tilde{H}$ on $\mathbf{U} \backslash \operatorname{int} \mathbf{K}_{f}$, i.e.,

$$
\begin{array}{llll}
\mathbf{U} \backslash \operatorname{int} \mathbf{K}_{f} & \stackrel{\tilde{H}}{\longrightarrow} & \tilde{\mathbf{U}} \backslash \operatorname{int} \mathbf{K}_{\tilde{f}} & \\
f \downarrow & & \downarrow \tilde{f} & \text { commutes. } \\
\mathbf{V} \backslash \operatorname{int} \mathbf{K}_{f} & \underset{\tilde{H}}{\longrightarrow} & \tilde{\mathbf{V}} \backslash \operatorname{int} \mathbf{K}_{\tilde{f}} &
\end{array}
$$

- We say that $f$ and $\tilde{f}$ are quasi-conformally conjugate if there is a (global) qc homeomorphism $H^{\prime}: \mathbf{V} \stackrel{\approx}{\underset{\mathbf{V}}{\longrightarrow}}$ so that $H^{\prime} \circ f=\tilde{f} \circ H^{\prime}$ on $\mathbf{U}$, i.e.,

$$
\begin{array}{rlll}
\mathbf{V} \supset \mathbf{U} & \stackrel{H^{\prime}}{\rightarrow} & \tilde{\mathbf{U}} \subset \tilde{\mathbf{V}} & \\
f \downarrow & & \downarrow \tilde{f} & \text { commutes. } \\
\mathbf{V} & \rightarrow & \tilde{\mathbf{V}} &
\end{array}
$$

The objective of rigidity is to show that some apparently weaker relations imply much stronger relations (such as qc conjugacy).

Definition. Let $f: \mathbf{U} \rightarrow \mathbf{V}$ be a proper box mapping.

(1) For every $n \geq 0$, we call each component of $f^{-n}(\mathbf{V})$ a puzzle piece of $\operatorname{depth} n$ for $f$. Denote by $\operatorname{depth}(P)$ the depth of a puzzle piece $P$.

We list below three basic properties of the puzzle pieces.

(a) Every puzzle piece is a quasi-disk and there are finitely many puzzle pieces of the same depth.

(b) Given two puzzle pieces $P$ and $Q$ with $\operatorname{depth}(P)>\operatorname{depth}(Q)$, either $P \subset \subset Q$ or $\bar{P} \cap \bar{Q}=\emptyset$.

(c) For $x \in \mathbf{K}_{f}$, for every $n \geq 0$, there is a unique puzzle piece of depth $n$ containing $x$. Denote the piece by $P_{n}(x)$. Then $P_{n+1}(x) \subset \subset P_{n}(x)$ and $\bigcap_{n \geq 0} P_{n}(x)$ is exactly the component of $\mathbf{K}_{f}$ containing $x$.

(2) Suppose that $X \subset \mathbf{V}$ is a finite union of puzzle pieces (not necessarily of the same depth). The set $X$ is called nice if for any $z \in \partial X$ and any $n \geq 1$, $f^{n}(z) \notin X$ as long as $f^{n}(z)$ is defined, that is, for any component $P$ of $X$, for any $n \geq 1, f^{n}(P)$ is not strictly contained in $X$. For example, if $X$ has a unique component, obviously it is a nice set.

Remark. 1. There are several kinds of puzzles, such as Yoccoz puzzle ([9, 28, 31]), Branner-Hubbard puzzle ([3]). The reader may refer to [23, 26, 27] for more information about the puzzles. The puzzles we exploit here are very similar to the Branner-Hubbard puzzles.

2. The definition of a nice set comes from [16].

Denote by Crit $(f)$ the set of critical points of $f$ and by

$$
\mathbf{P}:=\bigcup_{n \geq 1} \bigcup_{c \in \operatorname{Crit}(f)}\left\{f^{n}(c)\right\}
$$

the postcritical set of $f$.

For $x \in \mathbf{K}_{f}$, denote by $\mathbf{K}_{f}(x)$ the component of $\mathbf{K}_{f}$ containing the point $x$. We call a component of $\mathbf{K}_{f}$ a critical component if it contains a critical point. The map $f$ maps each component of $\mathbf{K}_{f}$ (resp. int $\mathbf{K}_{f}$ ) onto a component of $\mathbf{K}_{f}$ (resp. $\left.\operatorname{int} \mathbf{K}_{f}\right)$. A component $K$ of $\mathbf{K}_{f}\left(\right.$ resp. int $\left.\mathbf{K}_{f}\right)$ is called periodic if $f^{p}(K)=K$ for some $p \geq 1$, preperiodic if $f^{n}(K)$ is periodic for some $n \geq 0$, and wandering otherwise, that is, $f^{i}(K) \cap f^{j}(K)=\emptyset$ for all $i \neq j \geq 0$. 
We are now ready to state our main results.

Proposition 1.1 (quasi-conformally conjugate off $\mathbf{K}_{f}+(*)$ imply quasi-conformally conjugate off $\left.\operatorname{int} \mathbf{K}_{f}\right)$. Let $f: \mathbf{U} \rightarrow \mathbf{V}$ and $\tilde{f}: \tilde{\mathbf{U}} \rightarrow \tilde{\mathbf{V}}$ be two proper box mappings. Suppose that $f$ and $\tilde{f}$ are quasi-conformally conjugate off $\mathbf{K}_{f}$ by a qc map $H$. Assume furthermore that the following property (labeled by $(*)$ ) holds.

(*) There is a constant $\tilde{C}>0$, and for each pair $\left(n, \mathbf{K}_{f}(c)\right)$ with $n \geq 1$ and $\mathbf{K}_{f}(c)$ a critical component of $\mathbf{K}_{f}$, there exists a puzzle piece $Q_{n}(c)$ containing $c$ such that:

(i) the map $\left.H\right|_{\partial Q_{n}(c)}$ admits a $\tilde{C}$-qc extension inside $Q_{n}(c)$;

(ii) for a given critical component $\mathbf{K}_{f}(c)$, the pieces $\left\{Q_{n}(c)\right\}_{n \geq 1}$ form a nested sequence with $\bigcap_{n} Q_{n}(c)=\mathbf{K}_{f}(c)$ (the depth of $Q_{n}(c)$ may not be equal to $n$ );

(iii) for a given integer $n \geq 1$, the union $\bigcup_{c \in \operatorname{Crit}(f)} Q_{n}(c)$ forms a nice set.

Then the two maps $f, \tilde{f}$ are quasi-conformally conjugate off $\operatorname{int} \mathbf{K}_{f}$ by an extension of the map $H$.

Proposition 1.2. Let $f$ be a proper box mapping. Then $\operatorname{int}_{f}$ contains no wandering components.

Theorem 1.3 (quasi-conformally conjugate off $\mathbf{K}_{f}+(* *)$ imply quasi-conformally conjugate off $\left.\operatorname{int} \mathbf{K}_{f}\right)$. Let $f: \mathbf{U} \rightarrow \mathbf{V}$ and $\tilde{f}: \tilde{\mathbf{U}} \rightarrow \tilde{\mathbf{V}}$ be two proper box mappings. Suppose that $H: \mathbf{V} \backslash \mathbf{K}_{f} \rightarrow \tilde{\mathbf{V}} \backslash \mathbf{K}_{\tilde{f}}$ is a qc conjugacy off $\mathbf{K}_{f}$. Assume furthermore that the following assumption (labeled by $(* *)$ ) holds.

$(* *)$ Let $c$ be a critical point of $f$ belonging to a (pre)periodic component $\mathbf{K}_{f}(c)$ (of $\mathbf{K}_{f}$ ) whose periodic cycle contains a critical point. Denote by $P_{n}(c)$ the puzzle piece of depth $n$ containing $c$. There are a constant $M_{c}>0$ and an integer $N_{c} \geq 0$ such that for each $n \geq N_{c}$, the map $\left.H\right|_{\partial P_{n}(c)}$ admits an $M_{c^{-}} q c$ extension inside $P_{n}(c)$.

Then the map $H$ extends to a qc conjugacy off $\operatorname{int}_{f}$. Furthermore, if for every (pre)periodic component $K$ of $\mathbf{K}_{f}$ with nonempty interior, the map $\left.H\right|_{\partial K}$ extends to a qc conjugacy inside $K$, then $f$ and $\tilde{f}$ are quasi-conformally conjugate by an extension of $H$.

To see why the condition $(* *)$ is necessary one may consider two cubic polynomials $f$ and $\tilde{f}$ each having one escaping critical point at a given Böttcher position and the other critical point attracted by an attracting, resp., parabolic fixed point. Then $\mathbf{K}_{f}(c)$ is a quasi-circle and $\mathbf{K}_{\tilde{f}}(\tilde{c})$ is a cauliflower with inward cusps. These two polynomials are actually conformally conjugate on the basin of $\infty$, and topologically conjugate off int $\mathbf{K}_{f}$, but are clearly not quasi-conformally conjugate off $\operatorname{int} \mathbf{K}_{f}$.

Theorem 1.3 is the main result of this work. Notice that the assumption $(*)$ is a condition on uniform qc extensions of $H$ near EVERY critical component of $\mathbf{K}_{f}$, whereas the assumption $(* *)$ only requires such extensions around renormalizable critical components.

We will first prove that $(* *)$ implies $(*)$. This is the key ingredient and we will explain our strategy in more detail below. We then prove Proposition 1.1 using the well-known Spreading Principle (see [16]). This is done in Section 2. Actually, we will not directly apply this principle but use the proof of it in [16], with a slightly different presentation. We give a proof of Proposition 1.2 in Section 3. This section 
is self-contained, with an elementary surgery method that is quite different from the rest of the manuscript.

Finally, we combine Propositions 1.1 and 1.2 to complete the proof of Theorem 1.3 .

The key step that $(* *)$ implies $(*)$ is stated as the Main Proposition in Section 4. The proof of it is given in Section 5. In Section 5, we sometimes make use of the combinatorial tool - the tableau. This is an effective language invented by Branner and Hubbard ([3]) to deal with the complexity of the dynamics of puzzle pieces. To prove the Main Proposition, we will first make a classification of the critical points following their recurrence behavior. In order to get the uniformly qc extensions between the corresponding critical puzzle pieces of the two maps $f$ and $\tilde{f}$, we adapt the analytic method in [2, especially their Lemma 3.2 (stated as Lemma 5.9 below) to each class of critical points. Among all the classes, the most difficult one is the one containing persistently recurrent critical points (see Definition 2). To deal with those points, we resort to the enhanced nest constructed in $[16$. The nest is a sophisticated choice of puzzle pieces containing persistently recurrent critical points. In 23], the authors obtain some new estimates about the enhanced nest. Taking advantages of these estimates and Lemma 5.9. we can handle the persistently recurrent critical points.

In the appendix, we give an application of Theorem 1.3 . The main result in 4$]$ is to construct a holomorphic model for a multiply-connected fixed (super)attracting Fatou component of a rational map, and to show that such a model is unique. However, part of their unicity (rigidity) argument contains a gap. Here we will show how to use our Theorem 1.3 to fill in that gap.

\section{Proof of Proposition 1.1}

Suppose that $f$ and $\tilde{f}$ are quasi-conformally conjugate off $\mathbf{K}_{f}$ by a $C_{0}$-qc map $H$. Starting from the property $(*)$, we will prove that $H$ admits a qc extension across $\mathbf{K}_{f}$ and realizes a conjugacy off int $\mathbf{K}_{f}$.

Let $A$ be an open set and $z \in A$. Denote the component of $A$ containing $z$ by $\operatorname{Comp}_{z}(A)$. Given an open set $X$ consisting of finitely many puzzle pieces, let

$$
D(X)=\left\{z \in \mathbf{V} \mid \exists k \geq 0, f^{k}(z) \in X\right\}=\bigcup_{k \geq 0} f^{-k}(X) .
$$

For $z \in D(X) \backslash X$, let $k(z)$ be the minimal positive integer such that $f^{k(z)}(z) \in X$. Set

$$
\mathcal{L}_{z}(X):=\operatorname{Comp}_{z}\left(f^{-k(z)}\left(\operatorname{Comp}_{f(z)}(z)(X)\right)\right) .
$$

Obviously, $f^{k(z)}\left(\mathcal{L}_{z}(X)\right)=\operatorname{Comp}_{f^{k(z)}(z)}(X)$.

Lemma 2.1. Suppose that $X$ is a finite union of puzzle pieces. The following statements hold.

(1) For any $z \in D(X) \backslash X$, the sets $\mathcal{L}_{z}(X), f\left(\mathcal{L}_{z}(X)\right), \cdots, f^{k(z)-1}\left(\mathcal{L}_{z}(X)\right)$ are pairwise disjoint.

(2) Suppose that $X$ is nice and $z \in D(X) \backslash X$. Then for all $0 \leq i<k(z)$, we have $f^{i}\left(\mathcal{L}_{z}(X)\right) \cap X=\emptyset$. In particular, if $X \supset \operatorname{Crit}(f)$, then $\mathcal{L}_{z}(X)$ is conformally mapped onto a component of $X$ by $f^{k(z)}$. 
Proof. (1) Assume that there exist $0 \leq i<j<k(z)$ with $f^{i}\left(\mathcal{L}_{z}(X)\right) \cap f^{j}\left(\mathcal{L}_{z}(X)\right) \neq$ $\emptyset$. Then $f^{i}\left(\mathcal{L}_{z}(X)\right) \subset \subset f^{j}\left(\mathcal{L}_{z}(X)\right)$ and

$$
f^{k(z)-j}\left(f^{i}\left(\mathcal{L}_{z}(X)\right)\right) \subset \subset f^{k(z)-j}\left(f^{j}\left(\mathcal{L}_{z}(X)\right)\right)=f^{k(z)}\left(\mathcal{L}_{z}(X)\right)=\operatorname{Comp}_{f^{k(z)}(z)}(X) .
$$

So $f^{k(z)-j+i}(z) \in X$, but $0<k(z)-j+i<k(z)$. This is a contradiction with the minimality of $k(z)$.

(2) Assume that there is some $0 \leq i_{0}<k(z)$ with $f^{i_{0}}\left(\mathcal{L}_{z}(X)\right) \cap X \neq \emptyset$. We can show $f^{i_{0}}\left(\mathcal{L}_{z}(X)\right) \cap X \subset \subset f^{i_{0}}\left(\mathcal{L}_{z}(X)\right)$. In fact, when $i_{0} \neq 0$, this is due to the minimality of $k(z)$; when $i_{0}=0$, it is because $z \notin X$. Let $P$ be a component of $X$ with $P \subset \subset f^{i_{0}}\left(\mathcal{L}_{z}(X)\right)$. So $f^{k(z)-i_{0}}(P) \subset \subset f^{k(z)-i_{0}}\left(f^{i_{0}}\left(\mathcal{L}_{z}(X)\right)\right)=\operatorname{Comp}_{f^{k(z)}(z)}(X)$. It contradicts the condition that $X$ is nice.

The corollary below follows directly from the above lemma.

Corollary 2.2. Suppose that $X$ is a finite union of puzzle pieces. The following statements hold.

(i) For any $z \in D(X) \backslash X$, the set $\left\{\mathcal{L}_{z}(X), f\left(\mathcal{L}_{z}(X)\right), \cdots, f^{k(z)-1}\left(\mathcal{L}_{z}(X)\right)\right\}$ meets every critical point at most once and

$$
\operatorname{deg}\left(f^{k(z)}: \mathcal{L}_{z}(X) \rightarrow \operatorname{Comp}_{f^{k(z)}(z)}(X)\right) \leq\left(\max _{c \in \operatorname{Crit}(f)} \operatorname{deg}_{c}(f)\right)^{\# \operatorname{Crit}(f)}
$$

(ii) Suppose that $X$ is nice and $z \in D(X) \backslash X$. Then $\mathcal{L}_{w}(X)=\mathcal{L}_{z}(X)$ for all $w \in \mathcal{L}_{z}(X)$ and $\mathcal{L}_{w^{\prime}}(X) \cap \mathcal{L}_{z}(X)=\emptyset$ for all $w^{\prime} \notin \mathcal{L}_{z}(X)$.

(iii) Suppose that $X$ is nice and $z \in D(X) \backslash X$. Then $f^{i}\left(\mathcal{L}_{z}(X)\right)=\mathcal{L}_{f^{i}(z)}(X)$ for all $0<i<k(z)$.

Let $K$ be a critical component of $\mathbf{K}_{f}$ and $c_{1}, c_{2}, \cdots, c_{l}$ be all the critical points on $K$. Then $P_{n}\left(c_{1}\right)=P_{n}\left(c_{2}\right)=\cdots=P_{n}\left(c_{l}\right)$ and

$$
\operatorname{deg}\left(\left.f\right|_{P_{n}\left(c_{1}\right)}\right)=\left(\operatorname{deg}_{c_{1}}(f)-1\right)+\cdots+\left(\operatorname{deg}_{c_{l}}(f)-1\right)+1
$$

for all $n \geq 0$. We can view $K$ as a component containing one critical point of degree $\operatorname{deg}\left(\left.f\right|_{P_{n}\left(c_{1}\right)}\right)$. Hence in the following until the end of this article, we assume that each $\mathbf{V}$-component contains at most one critical point.

Now we will combine the property $(*)$ and the Spreading Principle to prove Proposition 1.1

Proof of Proposition 1.1. First fix $n \geq 1$. We shall follow the proof of the Spreading Principle in 16 to get a qc map $H_{n}$ from $\mathbf{V}$ onto $\tilde{\mathbf{V}}$.

Set $W_{n}:=\bigcup_{c \in \operatorname{Crit}(f)} Q_{n}(c)$. Then by Lemma 2.1 (2), each component of $D\left(W_{n}\right)$ is mapped conformally onto a component of $W_{n}$ by some iterate of $f$.

For every puzzle piece $P$, we can choose an arbitrary qc map $\phi_{P}: P \rightarrow \tilde{P}$ with $\left.\phi_{P}\right|_{\partial P}=\left.H\right|_{\partial P}$ since $H$ is a qc map from a neighborhood of $\partial P$ to a neighborhood of $\partial \tilde{P}$ and $\partial P, \partial \tilde{P}$ are quasi-circles (see e.g. [5], Lemma C.1). Note that by the definition of $W_{n}$, there are finitely many critical puzzle pieces not contained in $W_{n}$. So we can take $C_{n}^{\prime}$ to be an upper bound for the maximal dilatation of all the qc maps $\phi_{P}$, where $P$ runs over all puzzle pieces of depth 0 and all critical puzzle pieces not contained in $W_{n}$.

Given a puzzle piece $P$, let $0 \leq k \leq \operatorname{depth}(P)$ be the minimal nonnegative integer such that $f^{k}(P)$ is a critical puzzle piece or has depth 0. Set $\tau(P)=f^{k}(P)$. Then $f^{k}: P \rightarrow \tau(P)$ is a conformal map and so is $\tilde{f}^{k}: \tilde{P} \rightarrow \tau(\tilde{P})$, where $\tilde{P}$ is 
the puzzle piece bounded by $H(\partial P)$ for $\tilde{f}$ and $\tau(\tilde{P})=\tilde{f}^{k}(\tilde{P})$. Given a qc map $q: \tau(P) \rightarrow \tau(\tilde{P})$, we can lift it through the maps $f^{k}$ and $\tilde{f}^{k}$, that is, there is a qc map $p: P \rightarrow \tilde{P}$ such that $\tilde{f}^{k} \circ p=q \circ f^{k}$. Notice that the maps $p$ and $q$ have the same maximal dilatation, and if $\left.q\right|_{\partial \tau(P)}=\left.H\right|_{\partial \tau(P)}$, then $\left.p\right|_{\partial P}=\left.H\right|_{\partial P}$.

Let $Y_{0}=\mathbf{V}$ denote the union of all the puzzle pieces of depth 0 . Set $X_{0}=\emptyset$. For $j \geq 0$, we inductively define $X_{j+1}$ to be the union of puzzle pieces of depth $j+1$ such that each of these pieces is contained in $Y_{j}$ and is a component of $D\left(W_{n}\right)$; set $Y_{j+1}:=\left(Y_{j} \cap f^{-(j+1)}(\mathbf{V})\right) \backslash X_{j+1}$. We have the following relations: for any $j \geq 0$,

$Y_{j}=\left(Y_{j} \backslash f^{-(j+1)}(\mathbf{V})\right) \sqcup X_{j+1} \sqcup Y_{j+1}, \quad Y_{j+1} \subset \subset Y_{j}, \quad X_{j^{\prime}} \cap X_{j}=\emptyset$ for any $j^{\prime} \neq j$.

Given any component $Q$ of $Y_{j+1}$, we claim that $\tau(Q)$ is either one of the finitely many critical puzzle pieces not contained in $W_{n}$, or one of the finitely many puzzle pieces of depth 0 . In fact, for such $Q$, either $Q \cap D\left(W_{n}\right)=\emptyset$ or $Q \cap D\left(W_{n}\right) \neq \emptyset$. In the former case, since $\operatorname{Crit}(f) \subset W_{n} \subset D\left(W_{n}\right)$, the component $Q$ is mapped conformally onto a puzzle piece of depth 0 by $f^{\operatorname{depth}(Q)}$. So $\tau(Q)$ is a puzzle piece of depth 0 . In the latter case, if $Q \cap D\left(W_{n}\right) \subset \subset D\left(W_{n}\right)$, then $Q$ is compactly contained in a component of $D\left(W_{n}\right)$, denoted by $Q^{\prime}$, and $Q^{\prime} \subset \subset X_{j^{\prime}}$ for some $j^{\prime}<j+1$. But $Q \subset \subset Y_{j} \subset \subset Y_{j-1} \subset \subset \cdots \subset \subset Y_{0}$ and $X_{j} \cap Y_{j}=\emptyset, X_{j-1} \cap Y_{j-1}=\emptyset, \cdots, X_{0} \cap Y_{0}=\emptyset$. This is a contradiction. Hence $Q \cap D\left(W_{n}\right) \subset \subset Q$. If there is a critical point $c \in Q \cap D\left(W_{n}\right)$, then the component of $W_{n}$ containing $c$ is compactly contained in $Q$ and $\tau(Q)=Q$. Otherwise, the set $\tau(Q)$ must be a critical puzzle piece not contained in $W_{n}$.

Define $H^{(0)}=\phi_{P}$ on each component $P$ of $Y_{0}$. For each $j \geq 0$, assuming that $H^{(j)}$ is defined, we define $H^{(j+1)}$ as follows:

$$
H^{(j+1)}=\left\{\begin{array}{l}
H^{(j)} \\
H \\
\text { the univalent pullback of } \phi \\
\text { the univalent pullback of } \phi_{\tau(Q)}
\end{array}\right.
$$

on $\mathbf{V} \backslash Y_{j}$, on $Y_{j} \backslash f^{-(j+1)}(\mathbf{V})$, on each component of $X_{j+1}$, on each component $Q$ of $Y_{j+1}$,

where the map $\phi$ is the qc extension obtained by the assumption $(*)$.

Set $C_{n}=\max \left\{C_{0}, C_{n}^{\prime}, \tilde{C}\right\}$. The $\left\{H^{(j)}\right\}_{j \geq 0}$ is a sequence of $C_{n}$-qc maps. Hence it is precompact in the uniform topology.

By definition, $H^{(j)}=H^{(j+1)}$ outside $Y_{j}$. Thus, the sequence $\left\{H^{(j)}\right\}$ converges pointwise outside

$$
\bigcap_{j} Y_{j}=\left\{x \in \mathbf{K}_{f} \mid f^{k}(x) \notin W_{n}, k \geq 0\right\} .
$$

This set is a hyperbolic subset, on which $f$ is uniformly expanding, and hence has zero Lebesgue measure, in particular, no interior. So any two limit maps of the sequence $\left\{H^{(j)}\right\}_{j \geq 0}$ coincide on a dense open set of $\mathbf{V}$, therefore coincides on $\mathbf{V}$ to a unique limit map. Denote this map by $H_{n}$. It is $C_{n}$-qc.

By construction, $H_{n}$ coincides with $H$ on $\mathbf{V} \backslash\left(\left(\bigsqcup_{j} X_{j}\right) \cup\left(\bigcap_{j} Y_{j}\right)\right)$, is therefore $C_{0}$-qc there, and is $\tilde{C}$-qc on $\bigsqcup_{j} X_{j}$. It follows that the maximal dilatation of $H_{n}$ is bounded by $\max \left\{C_{0}, \tilde{C}\right\}$ except possibly on the set $\bigcap_{j} Y_{j}$, but this set has zero Lebesgue measure. It follows that the maximal dilatation of $H_{n}$ is $\max \left\{C_{0}, \tilde{C}\right\}$, which is independent of $n$. 
The sequence $H_{n}: \mathbf{V} \rightarrow \tilde{\mathbf{V}}$ has a subsequence converging uniformly to a limit

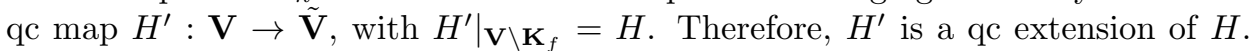
On the other hand, $H \circ f=\tilde{f} \circ H$ on $\mathbf{U} \backslash \mathbf{K}_{f}$. So $H^{\prime} \circ f=\tilde{f} \circ H^{\prime}$ holds on $\mathbf{U} \backslash \operatorname{int} \mathbf{K}_{f}$ by continuity. Therefore, $H^{\prime}$ is a qc conjugacy off $\operatorname{int} \mathbf{K}_{f}$. This ends the proof of Proposition 1.1

\section{Proof of Proposition 1.2}

In this section let $f: \mathbf{U} \rightarrow \mathbf{V}$ be a proper box mapping. Our strategy is to use some simple surgery to embed $\left(f, \mathbf{K}_{f}\right)$ into another dynamical system $\left(F, \mathbf{K}_{F}\right)$ (with possibly higher degree) which is conjugate to a polynomial, and then apply Sullivan's no-wandering-domain theorem.

Let $q \geq 1$ denote the number of components of $\mathbf{V}$. Enumerate the $\mathbf{V}$-components by $V_{1}, V_{2}, \cdots, V_{q}$.

Lemma 3.1. Let $\mathbf{W}$ be an open round disk centered at 0 with radius $>1$ containing $\overline{\mathbf{V}}$. The map $f: \mathbf{U} \rightarrow \mathbf{V}$ extends to a map $F$ on $\mathbf{V}$ so that:

-on each component $V_{i}$ of $\mathbf{V}$, the restriction $\left.F\right|_{V_{i}}: V_{i} \rightarrow \mathbf{W}$ is a quasi-regular branched covering;

- every component of $\mathbf{U}$ is a component of $F^{-1}(\mathbf{V})$;

-the restriction $F$ on $F^{-1}(\mathbf{V})$ is holomorphic.

Proof. Part I. Fix any component $V_{i}$ of $\mathbf{V}$ such that $V_{i} \cap \mathbf{U} \neq \emptyset$. We will extend $\left.f\right|_{V_{i} \cap \mathbf{U}}$ to a map $F$ on $V_{i}$ with the required properties. It will be done in three steps. Refer to Figure 2 for the construction of $F$ on $V_{i}$.
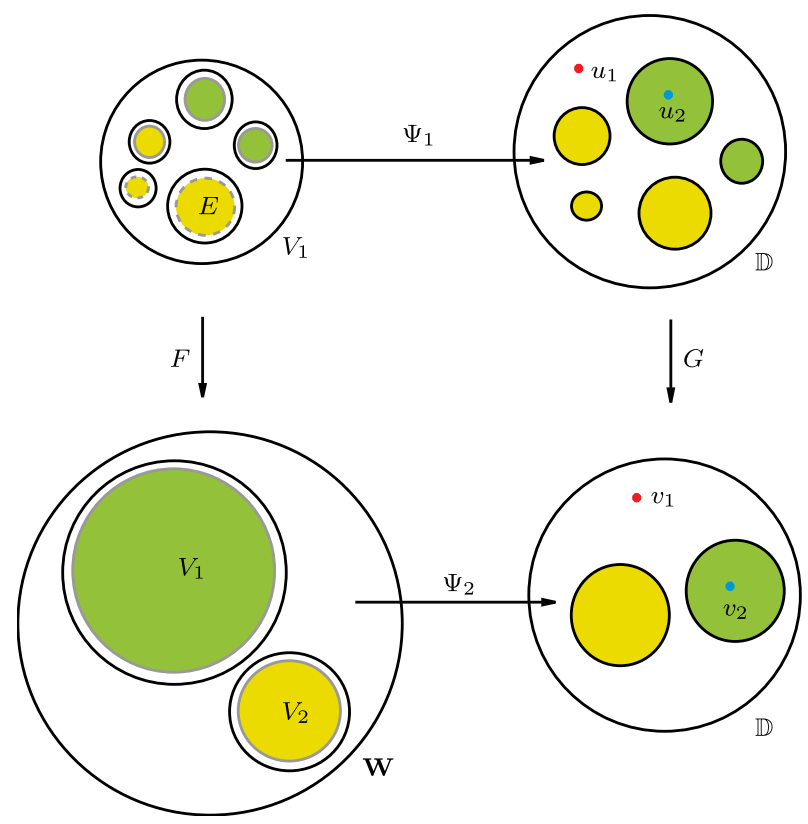

Figure 2. The construction of $F$ on $V_{1}$. In this figure, $q=2, i=1$. 
Step 1. The first step is to construct a Blaschke product $G: \mathbb{D} \rightarrow \mathbb{D}$ of degree $d_{i}$, where $\mathbb{D}$ denotes the unit disk and $d_{i}$ is determined below.

For every component $V_{j}$ of $\mathbf{V}$, we define

$$
q_{i j}=\#\left\{U \text { a component of } \mathbf{U} \mid U \subset V_{i}, f(U)=V_{j}\right\} .
$$

Set $q_{i}=\max _{j} q_{i j}$. Then $q_{i} \geq 1$ and

$$
\#\left\{\text { components of } \mathbf{U} \cap V_{i}\right\}=\sum_{j} q_{i j} \leq q \cdot q_{i} .
$$

We construct a Blaschke product $G: \mathbb{D} \rightarrow \mathbb{D}$, as well as a set $\mathcal{D}$ which is the union of $q$ Jordan domains in $\mathbb{D}$ with pairwise disjoint closures, as follows:

- If $V_{i}$ does not contain critical points of $f$, then set $G(z)=z^{q_{i}}$, and choose $\mathcal{D}$ to be a collection of $q$ Jordan domains compactly contained in $\mathbb{D} \backslash\{0\}$ with pairwise disjoint closures. Set $d_{i}=q_{i}$. Note that each component of $\mathcal{D}$ has exactly $q_{i}$ preimages. So

$$
\begin{aligned}
\#\left\{\text { components of } G^{-1}(\mathcal{D})\right\} & =q \cdot q_{i} \\
& \geq \#\left\{\text { components of } \mathbf{U} \cap V_{i}\right\} .
\end{aligned}
$$

- Otherwise, by the definition of a proper box mapping, the set $\operatorname{Crit}(f)$ intersects exactly one component $U$ of $\mathbf{U} \cap V_{i}$. Set $d_{i}=q_{i}+\operatorname{deg}\left(\left.f\right|_{U}\right)-1$. Choose $G$ so that it has degree $d_{i}$, and has two distinct critical points $u_{1}$ and $u_{2}$ such that $\operatorname{deg}_{u_{1}}(G)=q_{i}$, $\operatorname{deg}_{u_{2}}(G)=\operatorname{deg}\left(\left.f\right|_{U}\right)$ and $G\left(u_{1}\right) \neq G\left(u_{2}\right) 2$ Set $v_{i}=G\left(u_{i}\right), i=1,2$. Now choose $\mathcal{D}$ to be a collection of $q$ Jordan domains compactly contained in $\mathbb{D} \backslash\left\{v_{1}\right\}$ with pairwise disjoint closures and with $v_{2} \in \mathcal{D}$. Note that the preimage of any $\mathcal{D}$-component not containing $v_{2}$ has $d_{i}$ components, whereas the preimage of the $\mathcal{D}$-component containing $v_{2}$ has $d_{i}-\operatorname{deg}\left(\left.f\right|_{U}\right)+1=q_{i}$ components. So

$$
\begin{aligned}
\#\left\{\text { components of } G^{-1}(\mathcal{D})\right\} & =(q-1) d_{i}+q_{i} \\
& =(q-1)\left(q_{i}+\left.\operatorname{deg} f\right|_{U}-1\right)+q_{i} \\
& =q \cdot q_{i}+(q-1)\left(\left.\operatorname{deg} f\right|_{U}-1\right) \\
& >q \cdot q_{i} \\
& \geq \#\left\{\text { components of } \mathbf{U} \cap V_{i}\right\} .
\end{aligned}
$$

In both cases $G: \overline{\mathbb{D}} \backslash G^{-1}(\mathcal{D}) \rightarrow \overline{\mathbb{D}} \backslash \mathcal{D}$ is a proper map with a unique critical point.

Step 2. Make U, V 'thick'.

In $\mathbf{W}$, take $q$ Jordan domains with smooth boundaries $\widehat{V}_{j}, j=1, \cdots, q$, such that each $\widehat{V}_{j}$ is compactly contained in $\mathbf{W}, V_{j} \subset \widehat{V}_{j}$ for each $j=1, \cdots, q$, and all of the $\widehat{V}_{j}$ have pairwise disjoint closures. Denote $\widehat{\mathbf{V}}=\bigcup_{j=1}^{q} \widehat{V}_{j}$.

\footnotetext{
${ }^{2}$ One way to construct such a map $G$ is as follows: Consider the map $z \mapsto z^{q_{i}}$ together with a preimage $x \in] 0,1\left[\right.$ of $1 / 2$. Cut $\mathbb{D}$ along $\left[x, 1\right.$, glue in $\operatorname{deg}\left(\left.f\right|_{U}\right)$ consecutive sectors to define a new space $\tilde{D}$. Define a new map that maps each sector homeomorphically onto $\mathbb{D} \backslash\left[\frac{1}{2}, 1[\right.$, and agrees with $z \mapsto z^{q_{i}}$ elsewhere. This gives a branched covering $\hat{G}$ from $\tilde{D}$ onto $\mathbb{D}$ with two critical points and two critical values. Use $\hat{G}$ to pull back the standard complex structure of $\mathbb{D}$ turn $\tilde{D}$ in a Riemann surface. Uniformize $\tilde{D}$ by a map $\phi: \mathbb{D} \rightarrow \tilde{D}$. Then $G=\hat{G} \circ \phi$ suits what we need.
} 
In $V_{i}$, take $\widehat{\mathbf{U}}$ to be a union of \# $\left\{\right.$ components of $\left.G^{-1}(\mathcal{D})\right\}$ (which is greater than the number of $\mathbf{U}$-components in $V_{i}$ ) Jordan domains with smooth boundaries with the following properties:

- $\widehat{\mathbf{U}}$ is compactly contained in $V_{i}$;

- $\left(\mathbf{U} \cap V_{i}\right)$ is compactly contained in $\widehat{\mathbf{U}}$;

- each component of $\widehat{\mathbf{U}}$ contains at most one component of $\left(\mathbf{U} \cap V_{i}\right)$;

- the components of $\widehat{\mathbf{U}}$ have pairwise disjoint closures.

There exists a qc map $\Psi_{2}: \overline{\mathbf{W}} \rightarrow \overline{\mathbb{D}}$ such that $\Psi_{2}(\widehat{\mathbf{V}})=\mathcal{D}$.

Now, let $U$ be any component of $\mathbf{U}$. There is a unique component $\widehat{U}$ of $\widehat{\mathbf{U}}$ containing $U$. Also, $f(U)=V_{j} \subset \widehat{V}_{j}$ for some $j$, and $\Psi_{2}\left(\widehat{V}_{j}\right)$ is a component, denoted by $D(U)$, of $\mathcal{D}$. See the following diagram:

$$
\begin{array}{lcc}
U \subset \widehat{U} & & G^{-1}(D(U)) \\
\downarrow f & \downarrow G \\
V_{j} \subset \widehat{V}_{j} & \stackrel{\Psi_{2}}{\longrightarrow} & D(U)
\end{array}
$$

There is a qc map $\Psi_{1}: \bar{V}_{i} \rightarrow \overline{\mathbb{D}}$ so that $\Psi_{1}(\widehat{\mathbf{U}})=G^{-1}(\mathcal{D})$ and, for any component $U$ of $V_{i} \cap \mathbf{U}$, the set $\Psi_{1}(\widehat{U})$ is a component of $G^{-1}(D(U))$. Then we can define a quasi-regular branched covering $F: \bar{V}_{i} \backslash \widehat{\mathbf{U}} \rightarrow \overline{\mathbf{W}} \backslash \widehat{\mathbf{V}}$ of degree $d_{i}$ to be

$$
\left.\Psi_{2}^{-1} \circ G\right|_{\overline{\mathbb{D}} \backslash G^{-1}(\mathcal{D})} \circ \Psi_{1} \text {. }
$$

Step 3. Glue.

Define at first $F=f$ on $V_{i} \cap \mathbf{U}$. For each component $\widehat{E}$ of $\widehat{\mathbf{U}}$ not containing a component of $\mathbf{U}$, take a Jordan domain $E$ with smooth boundary compactly contained in $\widehat{E}$. Then $F$ maps $\partial \widehat{E}$ homeomorphically onto $\partial \widehat{V_{j}}$ for some $j$. Define $F$ to be a conformal map from $E$ onto $V_{j}$ by the Riemann Mapping Theorem and $F$ extends homeomorphically from $\bar{E}$ onto $\bar{V}_{j}$.

Notice that the map $F$ is defined everywhere except on a disjoint union of annular domains, one in each component of $\widehat{\mathbf{U}}$. Furthermore, $F$ maps the two boundary components of each such annular domain onto the boundary of $\widehat{V}_{j} \backslash V_{j}$ for some $j$, and is a covering of the same degree on each of the two boundary components.

This shows that $F$ admits an extension as a covering of these annular domains. As all boundary curves are smooth and $F$ is quasi-regular outside the annular domains, the extension can be made quasi-regular as well.

Part II. We may now extend $F$ to every $\mathbf{V}$ component intersecting $\mathbf{U}$ following the same procedure as shown in Part I. Assume that $V_{i}$ is a $\mathbf{V}$-component disjoint from $\mathbf{U}$. We define $F: V_{i} \rightarrow \mathbf{W}$ to be a conformal homeomorphism and we set $d_{i}=1$. We obtain a quasi-regular map $F: \mathbf{V} \rightarrow \mathbf{W}$ as an extension of $f: \mathbf{U} \rightarrow \mathbf{V}$. By construction, $F$ is holomorphic on $F^{-1}(\mathbf{V})$.

Lemma 3.2. There is an integer $d$ so that for the map $g: z \mapsto z^{d}$, the map $F$ has an extension on $\mathbf{W} \backslash \mathbf{V}$ so that $F: \mathbf{W} \backslash \mathbf{V} \rightarrow g(\mathbf{W}) \backslash \mathbf{W}$ is a quasi-regular branched covering, coincides with $g$ on $\partial \mathbf{W}$ and is continuous on $\overline{\mathbf{W}}$. In particular, $F^{-1}(\mathbf{W})=\mathbf{V}$ and $F$ is holomorphic on $F^{-2}(\mathbf{W})=F^{-1}(\mathbf{V})$.

Proof. Set $d=\sum_{i=1}^{q} d_{i}$, where the $d_{i}$ 's are defined in the proof of Lemma 3.1. See Figure 3 for the proof of this lemma.

The domain $\widehat{\mathbf{V}}$ is defined as in the proof of the previous lemma. Now take a Jordan domain $\widehat{\mathbf{W}}$ with smooth boundary such that $\mathbf{W} \subset \subset \widehat{\mathbf{W}} \subset \subset g(\mathbf{W})$. 

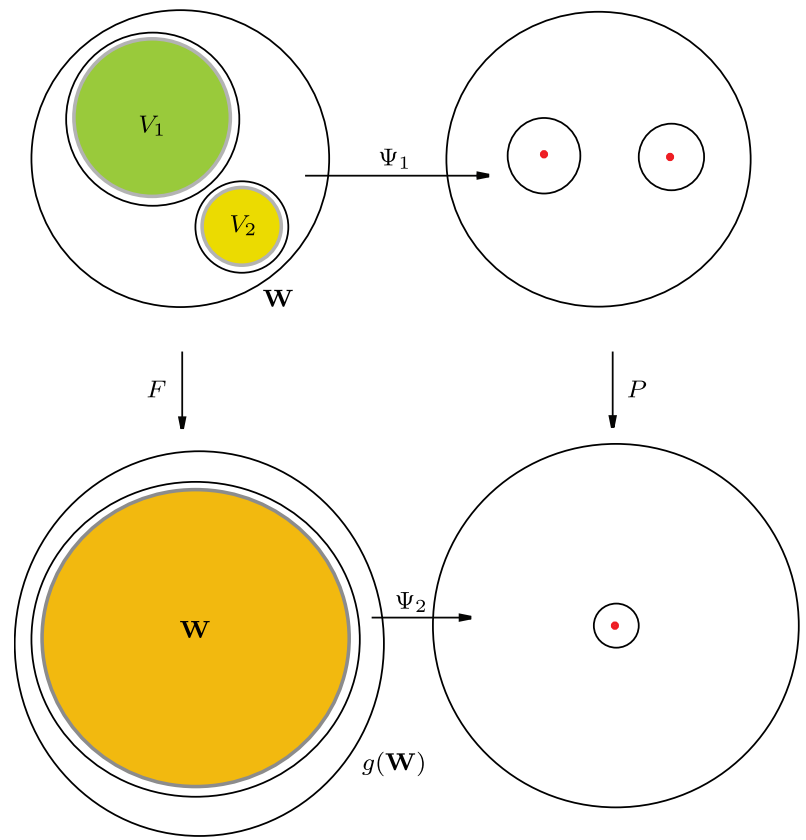

Figure 3

Let

$$
P(z)=\left(z-a_{1}\right)^{d_{1}}\left(z-a_{2}\right)^{d_{2}} \cdots\left(z-a_{q}\right)^{d_{q}},
$$

where $a_{1}, a_{2}, \cdots, a_{q} \in \mathbb{C}$ are distinct points.

Note that for each $1 \leq i \leq q$, we have $P\left(a_{i}\right)=0$, and $a_{i}$ is a critical point of $P$ whenever $d_{i}>1$.

Take $r>0$ small enough and $R>0$ large enough such that $\{0<|z| \leq r\} \cup\{R \leq$ $z \mid<\infty\}$ contains no critical value of $P$. Obviously $P: P^{-1}(\{r \leq|z| \leq R\}) \rightarrow\{r \leq$ $|z| \leq R\}$ is a holomorphic proper map of degree $d$.

Note that $P^{-1}\left(\{|z| \leq R\}\right.$ is a closed Jordan domain, and the set $P^{-1}(\{|z| \leq r\})$ consists of $q$ disjoint closed Jordan domains, each containing exactly one of the $a_{i}$ 's in the interior.

There exist qc maps $\Phi_{1}: \overline{\mathbf{W}} \rightarrow P^{-1}(\{|z| \leq R\}), \Phi_{2}: g(\overline{\mathbf{W}}) \rightarrow\{|z| \leq R\}$ such that for $i=1, \cdots, q$, the set $\Phi_{1}\left(\widehat{V}_{i}\right)$ is equal to the component of $P^{-1}(\{|z| \leq r\})$ containing $a_{i}$, and $\Phi_{2}(\widehat{\mathbf{W}})=\{|z| \leq r\}$, and

$$
P\left(\Phi_{1}(z)\right)=\Phi_{2}(g(z)), z \in \partial \mathbf{W}
$$

Set $F=\Phi_{2}^{-1} \circ P \circ \Phi_{1}$ on $\overline{\mathbf{W}} \backslash \widehat{\mathbf{V}}$.

Fix any $i=1, \cdots, q$. Both maps $F: \partial \widehat{V}_{i} \rightarrow \partial \widehat{\mathbf{W}}$ and $F: \partial V_{i} \rightarrow \partial \mathbf{W}$ are coverings of degree $d_{i}$. We may thus extend, as before, $F$ to a quasi-regular covering map from $\widehat{V}_{i} \backslash V_{i}$ onto $\widehat{\mathbf{W}} \backslash \mathbf{W}$.

This ends the construction of $F$. 
Proof of Proposition 1.2. Extend the map $F$ in Lemma 3.2 to $\overline{\mathbb{C}}$ by setting $F=g$ on $\overline{\mathbb{C}} \backslash \mathbf{W}$.

This $F$ is quasi-regular, and is holomorphic on $(\overline{\mathbb{C}} \backslash \mathbf{W}) \cup F^{-2}(\mathbf{W})$. So every orbit passes at most twice the region $\mathbf{W} \backslash F^{-2}(\mathbf{W})$. By the Surgery Principle (see Page 130 Lemma 15 in [1]), the map $F$ is quasi-conformally conjugate to a polynomial $h$. The set $\mathbf{K}_{F}$ can be defined as for $h$ and the two dynamical systems $\left.F\right|_{\mathbf{K}_{F}}$ and $\left.h\right|_{\mathbf{K}_{h}}$ are topologically conjugate.

Proposition 1.2 holds for the pair $\left(h, \mathbf{K}_{h}\right)$ (in place of $\left.\left(f, \mathbf{K}_{f}\right)\right)$ by Sullivan's nowandering-domain theorem. It follows that the result also holds for $\left(F, \mathbf{K}_{F}\right)$. But $\mathbf{K}_{f}$ is an $F$-invariant subset of $\mathbf{K}_{F}$ with every component of $\mathbf{K}_{f}$ being a component of $\mathbf{K}_{F}$, and with $\left.F\right|_{\mathbf{K}_{f}}=\left.f\right|_{\mathbf{K}_{f}}$. So the theorem holds for the pair $\left(f, \mathbf{K}_{f}\right)$.

Notice that one cannot expect to prove that $\left(f, \mathbf{K}_{f}\right)$ is itself topologically conjugate to a polynomial. Examples can be provided by taking the rational maps of degree $d$ constructed by Przytycki [25] such that they have a completely invariant Fatou domain containing less than $d-1$ critical points.

\section{Proof of Theorem 1.3}

Main Proposition $((* *)$ implies $(*))$. Let $f: \mathbf{U} \rightarrow \mathbf{V}$ and $\tilde{f}: \tilde{\mathbf{U}} \rightarrow \tilde{\mathbf{V}}$ be two proper box mappings. Suppose that $H: \mathbf{V} \backslash \mathbf{K}_{f} \rightarrow \tilde{\mathbf{V}} \backslash \mathbf{K}_{\tilde{f}}$ is a qc conjugacy off $\mathbf{K}_{f}$. We make the assumption $(* *)$ as in Theorem 1.3 . Then the property $(*)$ stated in Proposition 1.1 holds.

We will postpone the proof of the Main Proposition in the next section. Here we combine this proposition and Proposition 1.1 to give a proof of Theorem 1.3 .

Proof of Theorem 1.3. By the Main Proposition and Proposition 1.1, the qc conjugacy off $\mathbf{K}_{f} H$ extends to a qc conjugacy off int $\mathbf{K}_{f}$. By Proposition 1.2, every component of $\mathbf{K}_{f}$ with nonempty interior is preperiodic. Under the condition that for every preperiodic component $K$ of $\mathbf{K}_{f}$ with nonempty interior, the map $\left.H\right|_{\partial K}$ extends to a qc conjugacy inside $K$, we easily conclude that $H$ admits a qc extension across $\mathbf{K}_{f}$ such that $f$ and $\tilde{f}$ are quasi-conformally conjugate by this extension of $H$.

\section{Proof of Main Proposition}

In this section, we always assume that $f: \mathbf{U} \rightarrow \mathbf{V}$ is a proper box mapping such that any $\mathbf{V}$-component contains at most one critical point.

\subsection{Reduction of Main Proposition.}

Definition 1. (1) For $x, y \in \mathbf{K}_{f}$, we say that the forward orbit of $x$ combinatorially accumulates to $y$, written as $x \rightarrow y$, if for any $n \geq 0$, there is $j \geq 1$ such that $f^{j}(x) \in P_{n}(y)$. (This definition comes from [26].)

Clearly, if $x \rightarrow y$ and $y \rightarrow z$, then $x \rightarrow z$.

Let $\operatorname{Forw}(x)=\left\{y \in \mathbf{K}_{f} \mid x \rightarrow y\right\}$ for $x \in \mathbf{K}_{f}$.

(2) Define an equivalence relation in $\operatorname{Crit}(f)$ as follows:

$$
\text { for } c_{1}, c_{2} \in \operatorname{Crit}(f), c_{1} \sim c_{2} \Longleftrightarrow \text { either } c_{1}=c_{2} \text { or }\left(c_{1} \rightarrow c_{2} \text { and } c_{2} \rightarrow c_{1}\right) \text {. }
$$

Let $[c]$ denote the equivalence class containing $c$ for $c \in \operatorname{Crit}(f)$.

It is clear that $[c]=\{c\}$ if $c \not \rightarrow c$. 
(3) We say that $\left[c_{1}\right]$ accumulates to $\left[c_{2}\right]$, written as $\left[c_{1}\right] \rightarrow\left[c_{2}\right]$, if

$$
\exists c_{1}^{\prime} \in\left[c_{1}\right], \exists c_{2}^{\prime} \in\left[c_{2}\right] \text { such that } c_{1}^{\prime} \rightarrow c_{2}^{\prime} .
$$

It is easy to check that if $\left[c_{1}\right] \rightarrow\left[c_{2}\right]$, then

$$
\forall c_{1}^{\prime \prime} \in\left[c_{1}\right], \forall c_{2}^{\prime \prime} \in\left[c_{2}\right] \text { we have } c_{1}^{\prime \prime} \rightarrow c_{2}^{\prime \prime} .
$$

It follows from this property that if $\left[c_{1}\right] \rightarrow\left[c_{2}\right],\left[c_{2}\right] \rightarrow\left[c_{3}\right]$, then $\left[c_{1}\right] \rightarrow\left[c_{3}\right]$.

(4) Define $\mathcal{D}(f):=\operatorname{Crit}(f) / \sim$. Define a partial order $\leq$ in $\mathcal{D}(f)$ :

$$
\left[c_{1}\right] \leq\left[c_{2}\right] \Longleftrightarrow\left[c_{1}\right]=\left[c_{2}\right] \text { or }\left[c_{2}\right] \rightarrow\left[c_{1}\right] \text {. }
$$

We can decompose the quotient $\mathcal{D}(f)$ as follows. Let $\mathcal{D}_{0}(f)$ be the set of elements in $\mathcal{D}(f)$ which are minimal in the partial order $\leq$, that is, $[c] \in \mathcal{D}_{0}(f)$ if and only if $[c]$ does not accumulate to any element in $\mathcal{D}(f) \backslash\{[c]\}$. For every $k \geq 0$, assume that $\mathcal{D}_{k}(f)$ is defined, then $\mathcal{D}_{k+1}(f)$ is defined to be the set of elements in $\mathcal{D}(f)$ which are minimal in the set $\mathcal{D}(f) \backslash\left(\mathcal{D}_{k}(f) \cup \mathcal{D}_{k-1}(f) \cup \cdots \cup \mathcal{D}_{0}(f)\right)$ in the partial order $\leq$.

For the construction above, we prove the properties below (refer to Figure 4).

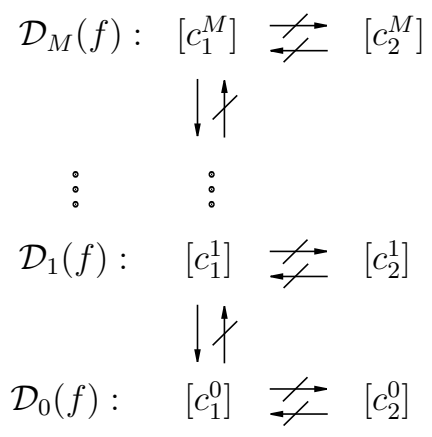

Figure 4

Lemma 5.1. (P1) There is an integer $M \geq 0$ such that $\mathcal{D}(f)=\bigsqcup_{k=0}^{M} \mathcal{D}_{k}(f)$.

(P2) For every $k \geq 0$, given $\left[c_{1}\right],\left[c_{2}\right] \in \mathcal{D}_{k}(f),\left[c_{1}\right] \neq\left[c_{2}\right]$, we have $\left[c_{1}\right] \not \rightarrow\left[c_{2}\right]$ and $\left[c_{2}\right] \not \rightarrow\left[c_{1}\right]$.

(P3) Let $\left[c_{1}\right] \in \mathcal{D}_{s}(f),\left[c_{2}\right] \in \mathcal{D}_{t}(f)$ with $s<t$. Then $\left[c_{1}\right] \not \rightarrow\left[c_{2}\right]$.

(P4) For every $k \geq 1$, every $[c]$ in $\mathcal{D}_{k}(f)$ accumulates to some element in $\mathcal{D}_{k-1}(f)$.

Proof. (P1) holds because $\mathcal{D}(f)$ is a finite set and $\mathcal{D}_{i}(f) \cap \mathcal{D}_{j}(f)=\emptyset$ for $i \neq j$.

(P2) and (P3) follow directly from the minimal property of the elements in $\mathcal{D}_{k}(f)$ for every $0 \leq k \leq M$.

(P4) Let $k=1$. If there is some element $\left[c_{1}\right] \in \mathcal{D}_{1}(f)$ such that it does not accumulate to any element in $\mathcal{D}_{0}(f)$, then combining with (P2) and (P3), we have that $\left[c_{1}\right]$ does not accumulate to any element in $\mathcal{D}(f) \backslash\left\{\left[c_{1}\right]\right\}$. Consequently, we know $\left[c_{1}\right] \in \mathcal{D}_{0}(f)$. But $\left[c_{1}\right] \in \mathcal{D}_{1}(f)$ and $\mathcal{D}_{0}(f) \cap \mathcal{D}_{1}(f)=\emptyset$ by $(\mathrm{P} 1)$. We get a contradiction. So any element in $\mathcal{D}_{1}(f)$ will accumulate to some element in $\mathcal{D}_{0}(f)$.

Now we suppose that $k \geq 2$ and $(\mathrm{P} 4)$ holds for $\mathcal{D}_{1}(f), \mathcal{D}_{2}(f), \cdots, \mathcal{D}_{k-1}(f)$. Assume that $(\mathrm{P} 4)$ is not true for $\mathcal{D}_{k}(f)$, that is, there is some $\left[c_{k}\right] \in \mathcal{D}_{k}(f)$ such that $\left[c_{k}\right]$ does not accumulate to any element in $\mathcal{D}_{k-1}(f)$. 
If $\left[c_{k}\right]$ does not accumulate to any element in $\bigcup_{j=0}^{k-2} \mathcal{D}_{j}(f)$, then by $(\mathrm{P} 2)$ and $(\mathrm{P} 3)$, we conclude that $\left[c_{k}\right] \in \mathcal{D}_{0}(f)$ which contradicts the condition that $\left[c_{k}\right] \in \mathcal{D}_{k}(f)$ and the fact that $\mathcal{D}_{k}(f) \cap \mathcal{D}_{0}(f)=\emptyset$ by $(\mathrm{P} 1)$.

Let $0 \leq i \leq k-2$ be an integer satisfying that $\left[c_{k}\right]$ does not accumulate to any element in $\bigcup_{j=i+1}^{k-1} \mathcal{D}_{j}(f)$ and $\left[c_{k}\right]$ accumulates to some element in $\mathcal{D}_{i}(f)$. Then $\left[c_{k}\right]$ will not accumulate to any element in $\bigcup_{j=i+1}^{M} \mathcal{D}_{j}(f) \backslash\left\{\left[c_{k}\right]\right\}$ and hence $\left[c_{k}\right] \in$ $\mathcal{D}_{i+1}(f)$. But notice that $i+1 \leq k-1$ and $\left[c_{k}\right] \in \mathcal{D}_{k}(f)$. A contradiction.

Combining with the transitive property stated in Definition 1 (3), we can consecutively apply (P4) above and prove the following.

Corollary 5.2. For every $k \geq 1$, every $[c]$ in $\mathcal{D}_{k}(f)$ accumulates to some element in $\mathcal{D}_{0}(f)$.

We will deduce the Main Proposition from the following result.

Proposition 5.3. Let $(f: \mathbf{U} \rightarrow \mathbf{V}),(\tilde{f}: \tilde{\mathbf{U}} \rightarrow \tilde{\mathbf{V}})$ be two proper box mappings. Suppose that $H: \mathbf{V} \backslash \mathbf{K}_{f} \rightarrow \tilde{\mathbf{V}} \backslash \mathbf{K}_{\tilde{f}}$ is a qc conjugacy off $\mathbf{K}_{f}$. We make the assumption $(* *)$ as in Theorem 1.3 . Then for every $c \in\left[c_{0}\right]$ and every integer $n \geq 1$, there is a puzzle piece $K_{n}(c)$ containing $c$ with the following properties.

(i) For every $c \in\left[c_{0}\right]$, the pieces $\left\{K_{n}(c)\right\}_{n \geq 1}$ are a nested sequence.

(ii) For each $n \geq 1, \bigcup_{c \in\left[c_{0}\right]} K_{n}(c)$ is a nice set.

(iii) There is a constant $\tilde{M}=\tilde{M}\left(\left[c_{0}\right]\right)$, such that for each $n \geq 1$ and each $c \in\left[c_{0}\right]$, $\left.H\right|_{\partial K_{n}(c)}$ admits an $\tilde{M}$-qc extension inside $K_{n}(c)$.

We will postpone the proof of Proposition 5.3 to the next subsection. Here we prove the following lemma and then use it and Proposition 5.3 to prove the Main Proposition.

Lemma 5.4. Let $\left[c_{1}\right]$ and $\left[c_{2}\right]$ be two distinct equivalence classes. Suppose that for each $i=1,2$, the set $W_{i}$ is a nice set consisting of finitely many puzzle pieces such that each piece contains a point in $\left[c_{i}\right]$.

(1) If $\left[c_{1}\right] \nrightarrow \rightarrow\left[c_{2}\right]$ and $\left[c_{2}\right] \nrightarrow \rightarrow\left[c_{1}\right]$, then $W_{1} \cup W_{2}$ is a nice set containing $\left[c_{1}\right] \cup\left[c_{2}\right]$.

(2) Suppose $\left[c_{2}\right] \not \rightarrow\left[c_{1}\right]$ and

$$
\min _{P_{2} \text { a comp. of } W_{2}} \operatorname{depth}\left(P_{2}\right) \geq \max _{P_{1} \text { a comp. of } W_{1}} \operatorname{depth}\left(P_{1}\right),
$$

i.e., the minimal depth of the components of $W_{2}$ is not less than the maximal depth of those of $W_{1}$. Then $W_{1} \cup W_{2}$ is nice.

Before proving this lemma, we need to give an assumption for simplicity. Notice that given two critical points $c, c^{\prime}$, if $c \not t c^{\prime}$, then there is some integer $n\left(c, c^{\prime}\right)$ depending on $c$ and $c^{\prime}$ such that for all $j \geq 1$, for all $n \geq n\left(c, c^{\prime}\right), f^{j}(c) \notin P_{n}\left(c^{\prime}\right)$. Since \#Crit $(f)<\infty$, we can take $n_{0}=\max \left\{n\left(c, c^{\prime}\right) \mid c, c^{\prime} \in \operatorname{Crit}(f)\right\}$. Without loss of generality, we may assume that $n_{0}=0$, that is to say, we assume that

$(* * *)$ for any two critical points $c, c^{\prime}$, for all $j \geq 1, f^{j}(c) \notin P_{0}\left(c^{\prime}\right)$ if $c \not \rightarrow c^{\prime}$.

In the following paragraphs until the end of this article, we always assume that $(* * *)$ holds. 
Proof of Lemma 5.4. (1) According to Definition 1 (3) and the assumption (***), we know that

$$
\begin{aligned}
{\left[c_{1}\right] \not \rightarrow\left[c_{2}\right] } & \Longleftrightarrow \forall c_{1}^{\prime} \in\left[c_{1}\right], \forall c_{2}^{\prime} \in\left[c_{2}\right], c_{1}^{\prime} \nrightarrow c_{2}^{\prime} \\
& \Longleftrightarrow \forall c_{1}^{\prime} \in\left[c_{1}\right], \forall c_{2}^{\prime} \in\left[c_{2}\right], \forall n \geq 0, \forall j \geq 0, f^{j}\left(c_{1}^{\prime}\right) \notin P_{n}\left(c_{2}^{\prime}\right) \\
& \Longleftrightarrow \text { for any puzzle piece } P \ni c_{1}^{\prime}, \forall n \geq 0, \forall j \geq 0, f^{j}(P) \cap P_{n}\left(c_{2}^{\prime}\right)=\emptyset .
\end{aligned}
$$

In particular, for any $c_{1}^{\prime} \in\left[c_{1}\right]$, any $c_{2}^{\prime} \in\left[c_{2}\right]$, for the component $P_{1}$ of $W_{1}$ containing $c_{1}^{\prime}$ and the component $P_{2}$ of $W_{2}$ containing $c_{2}^{\prime}$, for any $j \geq 0, f^{j}\left(P_{1}\right) \cap P_{2}=\emptyset$. It is equivalent to say that for any component $P$ of $W_{1}$, for any $j \geq 0, f^{j}(P) \cap W_{2}=\emptyset$.

Similarly, from the condition $\left[c_{2}\right] \not \rightarrow\left[c_{1}\right]$, we can conclude $f^{j}(Q) \cap W_{1}=\emptyset$ for any component $Q$ of $W_{2}$ and any $j \geq 0$. Hence $W_{1} \cup W_{2}$ is a nice set.

(2) On one hand, from the proof of (1), we know that the condition $\left[c_{2}\right] \not \rightarrow\left[c_{1}\right]$ implies $f^{j}(Q) \cap W_{1}=\emptyset$ for any component $Q$ of $W_{2}$ and any $j \geq 0$.

On the other hand, for any component $P$ of $W_{1}$, for any $j \geq 0$, we have

$$
\begin{aligned}
\operatorname{depth}\left(f^{j}(P)\right) & =\operatorname{depth}(P)-j \\
& \leq P_{1} \text { a comp. of } W_{1} \\
& \leq P_{2} \text { a comp. of } W_{2}
\end{aligned}
$$

and then $f^{j}(P)$ cannot be strictly contained in $W_{2}$.

Hence $W_{1} \cup W_{2}$ is nice.

Now we can derive the Main Proposition from Proposition 5.3 ,

Proof of the Main Proposition. (i) follows immediately from Proposition 5.3 (i).

(ii) For every $[\tilde{c}] \in \mathcal{D}(f)$ and every $\hat{c} \in[\tilde{c}]$, let $\left\{K_{n}(\hat{c})\right\}_{n \geq 1}$ be the puzzle pieces obtained in Proposition 5.3 ,

Given $\left[c_{0}\right] \in \mathcal{D}_{k}(f), 0 \leq k<M$, let $A_{k}\left(\left[c_{0}\right]\right)=\left\{[c] \in \mathcal{D}_{k+1}(f) \mid[c] \rightarrow\left[c_{0}\right]\right\}$. Clearly, \# $A_{k}\left(\left[c_{0}\right]\right)<\infty$.

Recall that $\mathcal{D}(f)=\bigsqcup_{i=0}^{M} \mathcal{D}_{i}(f)$. For every $\left[c_{0}\right] \in \mathcal{D}_{M}(f)$, set $Q_{n}(c)=K_{n}(c)$ for each $c \in\left[c_{0}\right]$.

Now consider $\left[c_{0}\right] \in \mathcal{D}_{M-1}(f)$.

If $A_{M-1}\left(\left[c_{0}\right]\right)=\emptyset$, then set $Q_{n}(c)=K_{n}(c)$ for each $c \in\left[c_{0}\right]$.

Otherwise, there exists a subsequence $\left\{l_{n}\right\}_{n \geq 1}$ of $\{n\}$ such that

$$
\min _{c^{\prime} \in\left[c_{0}\right]} \operatorname{depth}\left(K_{l_{n}}\left(c^{\prime}\right)\right) \geq \max _{\left[c^{\prime}\right] \in A_{M-1}\left(\left[c_{0}\right]\right)} \operatorname{depth}\left(Q_{n}\left(c^{\prime}\right)\right)
$$

because $\min _{c^{\prime} \in\left[c_{0}\right]} \operatorname{depth}\left(K_{n}\left(c^{\prime}\right)\right)$ increasingly tends to $\infty$ as $n \rightarrow \infty$.

We repeat this process consecutively to $\mathcal{D}_{M-2}(f), \cdots, \mathcal{D}_{0}(f)$ and then all $Q_{n}(c)$ are defined. Combining the properties (P2), (P3) stated in Lemma 5.1 and Lemma 5.4. we easily conclude that $\bigcup_{c \in \operatorname{Crit}(f)} Q_{n}(c)$ is a nice set for every $n \geq 1$.

(iii) Since $\# \mathcal{D}(f)<\infty$, we can take the constant $\tilde{C}=\max \{\tilde{M}([\tilde{c}]) \mid[\tilde{c}] \in$ $\mathcal{D}(f)\}$.

5.2. Proof of Proposition 5.3. First, we need to introduce a classification of the set $\operatorname{Crit}(f)$ and several preliminary results.

Definition 2. (i) Suppose $c \rightarrow c$. For $c_{1}, c_{2} \in[c]$, we say that the piece $P_{n+k}\left(c_{1}\right)$ is a child of $P_{n}\left(c_{2}\right)$ if $f^{k}\left(P_{n+k}\left(c_{1}\right)\right)=P_{n}\left(c_{2}\right)$ and $f^{k-1}: P_{n+k-1}\left(f\left(c_{1}\right)\right) \rightarrow P_{n}\left(c_{2}\right)$ is conformal. 
The critical point $c$ is called persistently recurrent if for every $n \geq 0$, every $c^{\prime} \in[c], P_{n}\left(c^{\prime}\right)$ has finitely many children. Otherwise, the critical point $c$ is said to be reluctantly recurrent. It is easy to check that if $c$ is persistently recurrent (resp. reluctantly recurrent), so is every $c^{\prime} \in[c]$.

(ii) Let

$$
\begin{aligned}
\operatorname{Crit}_{\mathrm{n}}(f) & =\left\{c \in \operatorname{Crit}(f) \mid c \nrightarrow \rightarrow c^{\prime} \text { for any } c^{\prime} \in \operatorname{Crit}(f)\right\}, \\
\operatorname{Crit}_{\mathrm{e}}(f) & =\left\{c \in \operatorname{Crit}(f) \mid c \nrightarrow c \text { and } \exists c^{\prime} \in \operatorname{Crit}(f) \text { such that } c \rightarrow c^{\prime}\right\}, \\
\operatorname{Crit}_{\mathrm{r}}(f) & =\{c \in \operatorname{Crit}(f) \mid c \rightarrow c \text { and } c \text { is reluctantly recurrent }\}, \\
\operatorname{Crit}_{\mathrm{p}}(f) & =\{c \in \operatorname{Crit}(f) \mid c \rightarrow c \text { and } c \text { is persistently recurrent }\} .
\end{aligned}
$$

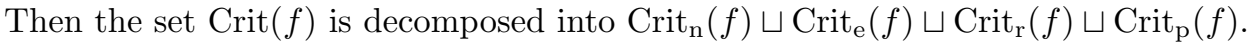

In this section, we will use sometimes the combinatorial tool - the tableau defined by Branner-Hubbard in 3]. The reader can also refer to [23, 26] for the definition of the tableau.

For $x \in \mathbf{K}_{f}$, the tableau $\mathcal{T}(x)$ is the graph embedded in $\left\{(u, v) \mid u \in \mathbb{R}^{-}, v \in \mathbb{R}\right\}$ with the axis of $u$ pointing downwards and the axis of $v$ pointing rightwards (this is the standard $\mathbb{R}^{2}$ with reversed orientation), with vertices indexed by $-\mathbb{N} \times \mathbb{N}$, where $\mathbb{N}=\{0,1, \cdots\}$, with the vertex at $(-m, 0)$ being $P_{m}(x)$, the puzzle piece of depth $m$ containing $x$, and with $f^{j}\left(P_{m}(x)\right)$ occupying the $(-m+j, j)$ th entry of $\mathcal{T}(x)$. The vertex at $(-m+j, j)$ is called critical if $f^{j}\left(P_{m}(x)\right)$ contains a critical point. If $f^{j}\left(P_{m}(x)\right)$ contains some $y \in \mathbf{K}_{f}$, we call the vertex at $(-m+j, j)$ a $y$-vertex.

All tableau satisfy the following three basic rules (see [3, 21, 23, 26]).

(Rule 1). In $\mathcal{T}(x)$ for $x \in \mathbf{K}_{f}$, if the vertex at $(-m, n)$ is a $y$-vertex, then so is the vertex at $(-i, n)$ for every $0 \leq i \leq m$.

(Rule 2). In $\mathcal{T}(x)$ for $x \in \mathbf{K}_{f}$, if the vertex at $(-m, n)$ is a $y$-vertex, then for every $0 \leq i \leq m$, the vertex at $(-m+i, n+i)$ is a vertex being $P_{-m+i}\left(f^{i}(y)\right)$.

(Rule 3) (See Figure 5). Given $x_{1}, x_{2} \in \mathbf{K}_{f}$. Suppose that there exist integers $m_{0} \geq 1, n_{0} \geq 0, i_{0} \geq 1, n_{1} \geq 1$ and critical points $c_{1}, c_{2}$ with the following properties.

(i) In $\mathcal{T}\left(x_{1}\right)$, the vertex at $\left(-\left(m_{0}+1\right), n_{0}\right)$ is a $c_{1}$-vertex and $\left(-\left(m_{0}+1-i_{0}\right), n_{0}+\right.$ $\left.i_{0}\right)$ is a $c_{2}$-vertex.

(ii) In $\mathcal{T}\left(x_{2}\right)$, the vertex at $\left(-m_{0}, n_{1}\right)$ is a $c_{1}$-vertex and $\left(-\left(m_{0}+1\right), n_{1}\right)$ is not critical.

If in $\mathcal{T}\left(x_{1}\right)$, for every $0<i<i_{0}$, the vertex at $\left(-\left(m_{0}-i\right), n_{0}+i\right)$ is not critical, then in $\mathcal{T}\left(x_{2}\right)$, the vertex at $\left(-\left(m_{0}+1-i_{0}\right), n_{1}+i_{0}\right)$ is not critical.

Recall that in Subsection 5.1, we made the assumption (***). Here, we translate that assumption in the language of the tableau. It is equivalent to assume that for $c, c^{\prime} \in \operatorname{Crit}(f), c^{\prime}$-vertex appears in $\mathcal{T}(c)$ iff $c^{\prime} \in \operatorname{Forw}(c)$.

Lemma 5.5. 1. Let $\mathbf{K}_{f}(c)$ be a periodic component of $\mathbf{K}_{f}$ with period $p$. Then the following properties hold:

(1) $f^{i}\left(\mathbf{K}_{f}\left(c^{\prime}\right)\right) \in\left\{\mathbf{K}_{f}(c), f\left(\mathbf{K}_{f}(c)\right), \cdots, f^{p-1}\left(\mathbf{K}_{f}(c)\right)\right\}, \forall c^{\prime} \in \operatorname{Forw}(c), \forall i \geq 0$.

(2) $\operatorname{Forw}(c)=[c]$.

(3) $c \in \operatorname{Crit}_{\mathrm{p}}(f)$.

2. Let $c \in \operatorname{Crit}_{\mathrm{p}}(f)$ with $\mathbf{K}_{f}(c)$ nonperiodic. Then the following properties hold:

(1) $\operatorname{Forw}(c)=[c]$.

(2) For every $c^{\prime} \in[c], c^{\prime} \in \operatorname{Crit}_{\mathrm{p}}(f)$ we have $\mathbf{K}_{f}\left(c^{\prime}\right)$ nonperiodic. 


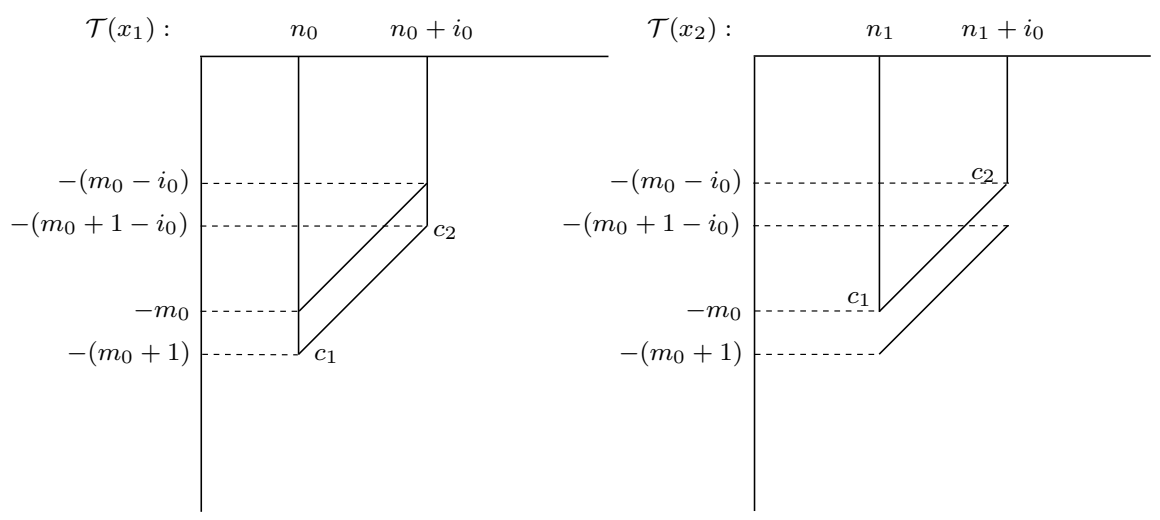

FIGURE 5

Proof. 1. Notice that $\mathbf{K}_{f}(c)$ is periodic iff there is a column in $\mathcal{T}(c) \backslash\{0$-th column $\}$ such that every vertex on that column is a $c$-vertex. According to this and using the tableau rules, it is easy to check that the statements in Point 1 are true.

2. (1) This property was proved by Qiu and Yin in Lemma 1, 26. For selfsufficiency, we repeat their proof here.

Assume that there is some $c^{\prime} \in \operatorname{Crit}(f)$ with $c \rightarrow c^{\prime}$ but $c^{\prime} \not \rightarrow c$. In the following, all the vertices we discuss are in $\mathcal{T}(c)$. One may refer to Figure 6 for the proof.

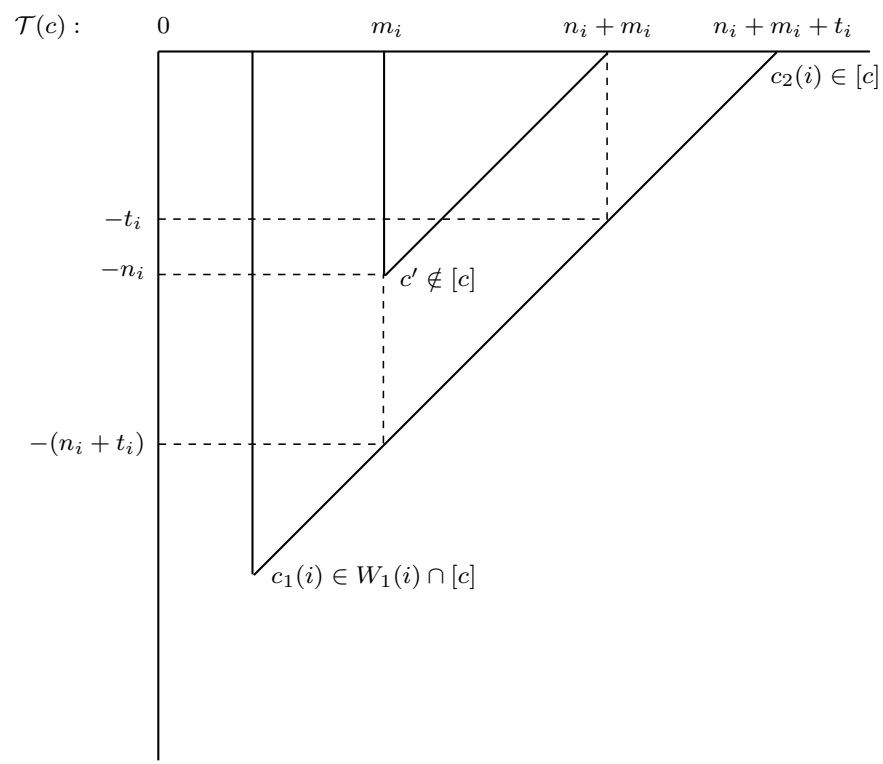

\section{FiguRE 6}

If there exists a column such that every vertex on it is a $c^{\prime}$-vertex, then $c^{\prime} \rightarrow c$ because $c \rightarrow c$. Hence there are infinitely many $c^{\prime}$-vertices $\left\{\left(-n_{i}, m_{i}\right)\right\}_{i \geq 1}$ such that $\left(-\left(n_{i}+1\right), m_{i}\right)$ is not critical and $\lim _{i \rightarrow \infty} n_{i}=\infty$. 
By the tableau rule (Rule 2) and the assumption (***), we can see that there are no vertices being critical points in $[c]$ on the diagonal starting from the vertex $\left(-n_{i}, m_{i}\right)$ and ending at the 0 -th row. Since $c \rightarrow c$, from the vertex $\left(0, n_{i}+m_{i}\right)$, one can march horizontally $t_{i} \geq 1$ steps to the right until the first hit of some $c_{2}(i)$-vertex in $[c]$. Then by (Rule 1 ), there are no vertices being critical points in $[c]$ on the diagonal from the vertex $\left(-t_{i}, n_{i}+m_{i}\right)$ to the vertex $\left(0, n_{i}+m_{i}+t_{i}\right)$. Therefore, there are no vertices being critical points in $[c]$ on the diagonal from the vertex $\left(-\left(n_{i}+t_{i}\right), m_{i}\right)$ to the vertex $\left(0, n_{i}+m_{i}+t_{i}\right)$, denote this diagonal by $I$.

If there exists a point $\tilde{c} \in \operatorname{Forw}(c) \backslash[c]$ on the diagonal $I$, then by the assumption $(* * *)$, every vertex, particularly the end vertex $\left(0, n_{i}+m_{i}+t_{i}\right)$ of $I$, cannot be a $\hat{c}$-vertex for any $\hat{c} \in[c]$. This contradicts the choice that the vertex $\left(0, n_{i}+m_{i}+t_{i}\right)$ is a $c_{2}(i)$-vertex for $c_{2}(i) \in[c]$.

Consequently, there are no critical points in Forw $(c)$ on the diagonal $I$. Combining with the assumption $(* * *)$, we know that there are no critical points on the diagonal $I$.

Follow the diagonal from the vertex $\left(-\left(n_{i}+t_{i}\right), m_{i}\right)$ left downwards until we reach a critical vertex $W_{1}(i)$ (such $W_{1}(i)$ exist since the 0 -th column vertex on that diagonal is critical). Let $c_{1}(i)$ be the critical point in $W_{1}(i)$. Then $c_{1}(i) \in[c]$ follows from the fact that $\left(0, n_{i}+m_{i}+t_{i}\right)$ is a $c_{2}(i)$-vertex for $c_{2}(i) \in[c]$ and the assumption $(* * *)$.

Therefore, $W_{1}(i)$ is a child of $P_{0}\left(c_{2}(i)\right)$. Notice that the depth of $W_{1}(i)$ is greater than $n_{i}$. As $c_{2}(i)$ lives in the finite set $[c]$ and $n_{i} \rightarrow \infty$ when $i \rightarrow \infty$, some point in $[c]$ must have infinitely many children. This is a contradiction with the condition $c \in \operatorname{Crit}_{p}(f)$.

(2) follows directly from Point 1 (1) and Point 1 (2).

Set

$$
\operatorname{Crit}_{\text {per }}(f)=\left\{c \in \operatorname{Crit}_{\mathrm{p}}(f) \mid \mathbf{K}_{f}(c) \text { is periodic }\right\} .
$$

Lemma 5.6. (i) If $c \in \operatorname{Crit}_{\mathrm{n}}(f) \cup \operatorname{Crit}_{\mathrm{p}}(f)$, then $[c] \in \mathcal{D}_{0}(f)$; if $c \in \operatorname{Crit}_{\mathrm{e}}(f)$, then $[c] \notin \mathcal{D}_{0}(f)$.

(ii) For every $c_{0} \in \operatorname{Crit}(f)$, exactly one of the following cases occurs.

Case 1. $\operatorname{Forw}\left(c_{0}\right) \cap\left(\operatorname{Crit}_{\mathrm{n}}(f) \cup \operatorname{Crit}_{\mathrm{r}}(f)\right) \neq \emptyset$.

Case 2. $\operatorname{Forw}\left(c_{0}\right) \subset \operatorname{Crit}_{\mathrm{p}}(f)$.

Case 3. For any $c \in \operatorname{Forw}\left(c_{0}\right)$, either $(\mathrm{a}): c \in \operatorname{Crit}_{\mathrm{p}}(f)$ or $(\mathrm{b}): c \in \operatorname{Crit}_{\mathrm{e}}(f)$ and Forw $(c)$ contains a critical point in $\operatorname{Crit}_{\mathrm{p}}(f)$; the critical point in (b) always exists.

Proof. (i) By the definitions of $\operatorname{Crit}_{\mathrm{n}}(f)$ and $\operatorname{Crit}_{\mathrm{e}}(f)$, we easily see that if $c \in$ $\operatorname{Crit}_{\mathrm{n}}(f),[c]=\{c\} \in \mathcal{D}_{0}(f)$ and if $c \in \operatorname{Crit}_{\mathrm{e}}(f),[c]=\{c\} \notin \mathcal{D}_{0}(f)$. If $c \in \operatorname{Crit}_{\mathrm{p}}(f)$, then by the previous lemma, we know that $\operatorname{Forw}(c)=[c]$ and then $[c] \in \mathcal{D}_{0}(f)$.

(ii) Suppose that neither Case 1 nor Case 2 happens. Let $c \in \operatorname{Forw}\left(c_{0}\right)$ with $c \notin \operatorname{Crit}_{\mathrm{p}}(f)$. Notice that $\operatorname{Crit}(f)=\operatorname{Crit}_{\mathrm{n}}(f) \cup \operatorname{Crit}_{\mathrm{r}}(f) \cup \operatorname{Crit}_{\mathrm{p}}(f) \cup \operatorname{Crit}_{\mathrm{e}}(f)$. So $c \in \operatorname{Crit}_{\mathrm{e}}(f)$ and then by (i), $[c] \in \mathcal{D}_{k}(f)$ for some $k \geq 1$. It follows from Corollary 5.2 that $[c]=\{c\}$ accumulates to some element $[\tilde{c}] \in \mathcal{D}_{0}(f)$.

Since Case 1 does not happen, we conclude that for every $[\hat{c}] \in \mathcal{D}_{0}(f)$ with $\left[c_{0}\right] \rightarrow$ $[\hat{c}]$, every point in $[\hat{c}]$ belongs to $\operatorname{Crit}_{\mathrm{p}}(f)$. Note that $\left[c_{0}\right] \rightarrow[c] \rightarrow[\tilde{c}]$ and $[\tilde{c}] \in \mathcal{D}_{0}(f)$. Hence every point in $[\tilde{c}]$ belongs to $\operatorname{Crit}_{\mathrm{p}}(f)$, particularly, $\tilde{c} \in \operatorname{Crit}_{\mathrm{p}}(f)$. 
Recall that in Section 2, for an open set $X$ consisting of finitely many puzzle pieces, we have defined the sets $D(X)$ and $\mathcal{L}_{z}(X)$ for $z \in D(X) \backslash X$. The following is a property about $\mathcal{L}_{z}(X)$ when $X$ consists of a single piece.

Lemma 5.7. Let $P$ be a puzzle piece and let the set $\left\{x_{1}, \cdots, x_{m}\right\} \subset \mathbf{V}$ be a finite set of points with each $x_{i} \in D(P) \backslash P$ for $1 \leq i \leq m$. Let $f^{k_{i}}\left(\mathcal{L}_{x_{i}}(P)\right)=P$ for some $k_{i} \geq 1$. Then

(1) for every $1 \leq i \leq m$, every $0 \leq j<k_{i}$, either

$$
f^{j}\left(\mathcal{L}_{x_{i}}(P)\right)=\mathcal{L}_{x_{s}}(P) \text { for some } 1 \leq s \leq m
$$

or

$$
f^{j}\left(\mathcal{L}_{x_{i}}(P)\right) \cap \mathcal{L}_{x_{t}}(P)=\emptyset \text { for all } 1 \leq t \leq m
$$

(2) $\bigcup_{i=1}^{m} \mathcal{L}_{x_{i}}(P) \cup P$ is a nice set.

Proof. (1) (by contradiction). Assume that there are integers $1 \leq i_{0} \leq m, 0 \leq j_{0}<$ $k_{i_{0}}$, and there are some $\mathcal{L}_{x_{i_{1}}}(P)$ for $1 \leq i_{1} \leq m$, such that

$$
f^{j_{0}}\left(\mathcal{L}_{x_{i_{0}}}(P)\right) \neq \mathcal{L}_{x_{i_{1}}}(P) \text { and } f^{j_{0}}\left(\mathcal{L}_{x_{i_{0}}}(P)\right) \cap \mathcal{L}_{x_{i_{1}}}(P) \neq \emptyset .
$$

Then either $f^{j_{0}}\left(\mathcal{L}_{x_{i_{0}}}(P)\right) \subset \subset \mathcal{L}_{x_{i_{1}}}(P)$ or $f^{j_{0}}\left(\mathcal{L}_{x_{i_{0}}}(P)\right) \supset \supset \mathcal{L}_{x_{i_{1}}}(P)$.

We may assume $f^{j_{0}}\left(\mathcal{L}_{x_{i_{0}}}(P)\right) \subset \subset \mathcal{L}_{x_{i_{1}}}(P)$. The proof of the other case is similar.

On one hand, since $f^{k_{i_{0}}-j_{0}}$ maps $f^{j_{0}}\left(\mathcal{L}_{x_{i_{0}}}(P)\right)$ onto $P$ and $f^{k_{i_{1}}}$ maps $\mathcal{L}_{x_{i_{1}}}(P)$ onto $P$, we have $k_{i_{0}}-j_{0}>k_{i_{1}}$.

On the other hand, by Corollary $2.2(3)$, we know that $f^{j_{0}}\left(\mathcal{L}_{x_{i_{0}}}(P)\right)=\mathcal{L}_{f^{j_{0}}\left(x_{i_{0}}\right)}(P)$ and then $k_{i_{0}}-j_{0}$ is the first landing time of the points in $f^{j_{0}}\left(\mathcal{L}_{x_{i_{0}}}(P)\right)$ to $P$, while from the assumption $f^{j_{0}}\left(\mathcal{L}_{x_{i_{0}}}(P)\right) \subset \subset \mathcal{L}_{x_{i_{1}}}(P)$, we have that $k_{i_{1}}$ is also the first landing time of the points in $f^{j_{0}}\left(\mathcal{L}_{x_{i_{0}}}(P)\right)$ to $P$. So $k_{i_{0}}-j_{0}=k_{i_{1}}$. A contradiction.

(2) For any $q \geq 1$ (as long as $\operatorname{depth}\left(f^{q}(P)\right) \geq 0$ ),

$$
\operatorname{depth}\left(f^{q}(P)\right)<\operatorname{depth}(P)<\operatorname{depth}\left(\mathcal{L}_{x_{s}}(P)\right)
$$

for every $1 \leq s \leq m$. So $f^{q}(P)$ cannot be strictly contained in $\bigcup_{i=1}^{m} \mathcal{L}_{x_{i}}(P)$ for all $q \geq 1$.

Fix $1 \leq i \leq m$. For $1 \leq j<k_{i}$, by $(1)$, we know that $f^{j}\left(\mathcal{L}_{x_{i}}(P)\right)$ is not strictly contained in $\bigcup_{i=1}^{m} \mathcal{L}_{x_{i}}(P)$. Since $P$ is a single puzzle piece, it is nice. By Lemma 2.1 (2), we have $f^{j}\left(\mathcal{L}_{x_{i}}(P)\right) \cap P=\emptyset$. When $j \geq k_{i}$, notice that as long as $\operatorname{depth}\left(f^{j}\left(\mathcal{L}_{x_{i}}(P)\right)\right) \geq 0$, we have

$$
\operatorname{depth}\left(f^{j}\left(\mathcal{L}_{x_{i}}(P)\right)\right) \leq \operatorname{depth}(P)<\operatorname{depth}\left(\mathcal{L}_{x_{s}}(P)\right)
$$

for every $1 \leq s \leq m$. This implies that $f^{j}\left(\mathcal{L}_{x_{i}}(P)\right)$ is not strictly contained in $\bigcup_{i=1}^{m} \mathcal{L}_{x_{i}}(P) \cup P$.

Lemma 5.8. Let $Q, Q^{\prime}, P, P^{\prime}$ be puzzle pieces with the following properties:

(a) $Q \subset \subset Q^{\prime}, c_{0} \in P \subset \subset P^{\prime}$ for $c_{0} \in \operatorname{Crit}(f)$.

(b) There is an integer $l \geq 1$ such that $f^{l}(Q)=P, f^{l}\left(Q^{\prime}\right)=P^{\prime}$.

(c) $\left(P^{\prime} \backslash P\right) \cap\left(\bigcup_{c \in \text { Forw }\left(c_{0}\right)} \bigcup_{n \geq 0}\left\{f^{n}(c)\right\}\right)=\emptyset$.

Then for all $0 \leq i \leq l,\left(f^{i}\left(Q^{\prime}\right) \backslash f^{i}(Q)\right) \cap \operatorname{Forw}\left(c_{0}\right)=\emptyset$.

Proof. If $f^{l-1}\left(Q^{\prime}\right) \backslash f^{l-1}(Q)$ contains some $c \in$ Forw $\left(c_{0}\right)$, since $f\left(f^{l-1}(Q)\right)=P$ and $\operatorname{deg}\left(f: f^{l-1}\left(Q^{\prime}\right) \rightarrow P^{\prime}\right)=\operatorname{deg}_{c}(f)$, we have $f(c) \in P^{\prime} \backslash P$. This contradicts condition (c).

For the case $l=1$, the lemma holds. 
Now assume $l \geq 2$.

We first prove $\left(f^{l-2}\left(Q^{\prime}\right) \backslash f^{l-2}(Q)\right) \cap \operatorname{Forw}\left(c_{0}\right)=\emptyset$.

If $\left(f^{l-1}\left(Q^{\prime}\right) \backslash f^{l-1}(Q)\right) \cap\left(\operatorname{Crit}(f) \backslash \operatorname{Forw}\left(c_{0}\right)\right)=\emptyset$, then

$$
f: f^{l-1}\left(Q^{\prime}\right) \backslash f^{l-1}(Q) \rightarrow P^{\prime} \backslash P .
$$

If $f^{l-2}\left(Q^{\prime}\right) \backslash f^{l-2}(Q)$ contains some $c^{\prime} \in$ Forw $\left(c_{0}\right)$, since $f\left(f^{l-2}(Q)\right)=f^{l-1}(Q)$ and $\operatorname{deg}\left(f: f^{l-2}\left(Q^{\prime}\right) \rightarrow f^{l-1}\left(Q^{\prime}\right)\right)=\operatorname{deg}_{c^{\prime}}(f)$, we have $f\left(c^{\prime}\right) \in f^{l-1}\left(Q^{\prime}\right) \backslash f^{l-1}(Q)$ and $f^{2}\left(c^{\prime}\right) \in P^{\prime} \backslash P$. This contradicts condition (c). Hence under the assumption $\left(f^{l-1}\left(Q^{\prime}\right) \backslash f^{l-1}(Q)\right) \cap\left(\operatorname{Crit}(f) \backslash\right.$ Forw $\left.\left(c_{0}\right)\right)=\emptyset$, we come to the conclusion that $\left(f^{l-2}\left(Q^{\prime}\right) \backslash f^{l-2}(Q)\right) \cap$ Forw $\left(c_{0}\right)=\emptyset$.

Otherwise, $f^{l-1}\left(Q^{\prime}\right) \backslash f^{l-1}(Q)$ contains some $c_{1} \in \operatorname{Crit}(f) \backslash$ Forw $\left(c_{0}\right)$. Since $c_{1} \notin$ Forw $\left(c_{0}\right), c_{1} \notin \operatorname{Forw}(c)$ for any $c \in \operatorname{Forw}\left(c_{0}\right)$. By the assumption $(* * *)$, we conclude that $\left(f^{l-2}\left(Q^{\prime}\right) \backslash f^{l-2}(Q)\right) \cap \operatorname{Forw}\left(c_{0}\right)=\emptyset$.

Continue the similar argument as above, we prove the lemma for all $0 \leq i \leq$ $l-3$.

The analytic method we will use to prove Proposition 5.3 is the following lemma on covering maps of the unit disk.

Lemma 5.9 (see [2, Lemma 3.2]). For every integer $d \geq 2$ and every $0<\rho<r<1$ there exists $L_{0}=L_{0}(\rho, r, d)$ with the following property. Let $g, \tilde{g}:(\mathbb{D}, 0) \rightarrow(\mathbb{D}, 0)$ be holomorphic proper maps of the same degree at most $d$, with critical values contained in $\mathbb{D}_{\rho}$. Let $\eta, \eta^{\prime}: \mathbb{T} \rightarrow \mathbb{T}$ be two homeomorphisms satisfying $\tilde{g} \circ \eta^{\prime}=\eta \circ g$, where $\mathbb{T}$ denotes the unit circle. Assume that $\eta$ admits an $L$-qc extension $\xi: \mathbb{D} \rightarrow \mathbb{D}$ which is the identity on $\mathbb{D}_{r}$. Then $\eta^{\prime}$ admits an $L^{\prime}$-qc extension $\xi^{\prime}: \mathbb{D} \rightarrow \mathbb{D}$ which is the identity on $\mathbb{D}_{r}$, where $L^{\prime}=\max \left\{L, L_{0}\right\}$.

In the following, we will discuss $\operatorname{Crit}_{\text {per }}(f), \operatorname{Crit}_{\mathrm{p}}(f), \operatorname{Crit}_{\mathrm{n}}(f) \cup \mathrm{Crit}_{\mathrm{r}}(f)$ and $\mathrm{Crit}_{\mathrm{e}}(f)$ successively.

For any $c \in \mathrm{Crit}_{\text {per }}(f)$, by the assumption $(* *)$ in Proposition [5.3, there are a constant $M_{c}$ and an integer $N_{c}$ such that the map $\left.H\right|_{\partial P_{n}(c)}$ extends to an $M_{c}$-qc extension inside $P_{n}(c)$ for all $n \geq N_{c}$.

The following lemma can be easily proved by Lemma 5.7 .

Lemma 5.10. Fix a point $c_{0} \in \operatorname{Crit}_{\text {per }}(f)$ and set $N:=\max \left\{N_{c}, c \in\left[c_{0}\right]\right\}$. Let $K_{n}\left(c_{0}\right)=P_{n+N}\left(c_{0}\right)$ and for every $c \in\left[c_{0}\right] \backslash\left\{c_{0}\right\}$, let $K_{n}(c)=P_{n+N+l_{c}}(c)$, where $l_{c}$ is the smallest positive integer such that $f^{l_{c}}\left(\mathbf{K}_{f}(c)\right)=\mathbf{K}_{f}\left(c_{0}\right)$. Then $\bigcup_{c \in\left[c_{0}\right]} K_{n}(c)$ is nice for every $n \geq 1$.

Set $b=\# \operatorname{Crit}(f), \delta=\max _{c \in \operatorname{Crit}(f)} \operatorname{deg}_{c}(f)$, and

$$
\operatorname{orb}_{f}\left(\left[c_{0}\right]\right)=\bigcup_{n \geq 0} \bigcup_{c \in\left[c_{0}\right]}\left\{f^{n}(c)\right\}
$$

for $c_{0} \in \operatorname{Crit}(f)$.

The following theorem was originally proved by Qiu-Yin [26] and by Kozlovskivan Strien [17] independently. It is restated in this form in 23. The proof uses the enhanced nest ([16]) and the Kahn-Lyubich covering lemma ([14]).

Theorem 5.11. Given a critical point $c_{0} \in \operatorname{Crit}_{\mathrm{p}}(f) \backslash \operatorname{Crit}_{\text {per }}(f)$. There are two constants $S$ and $\Delta_{0}>0$, depending on $b, \delta$ and $\widehat{\mu}$ (see below), and a nested sequence of critical puzzle pieces $K_{n}\left(c_{0}\right) \subset \subset K_{n-1}\left(c_{0}\right), n \geq 1$, with $K_{0}\left(c_{0}\right)$ to be the critical 
puzzle piece of depth 0 , satisfying that

(i) for each $K_{n}\left(c_{0}\right), n \geq 1$, we have $f^{p_{n}}\left(K_{n}\left(c_{0}\right)\right)=K_{n-1}\left(c_{0}\right)$ for some $p_{n} \geq 1$ and $\operatorname{deg}\left(f^{p_{n}}: K_{n}\left(c_{0}\right) \rightarrow K_{n-1}\left(c_{0}\right)\right) \leq S$,

(ii) each $K_{n}\left(c_{0}\right), n \geq 1$, contains a sub-critical piece $K_{n}^{-}\left(c_{0}\right)$ such that

$$
\bmod \left(K_{n}\left(c_{0}\right) \backslash \overline{K_{n}^{-}\left(c_{0}\right)}\right) \geq \Delta_{0} \text { and }\left(K_{n}\left(c_{0}\right) \backslash \overline{K_{n}^{-}\left(c_{0}\right)}\right) \cap \operatorname{orb}_{f}\left(\left[c_{0}\right]\right)=\emptyset .
$$

Here

$$
\widehat{\mu}=\min \left\{\bmod \left(P_{0}\left(c_{0}\right) \backslash \bar{W}\right) \mid W \text { a component of } \mathbf{U} \text { contained in } P_{0}\left(c_{0}\right)\right\} \text {. }
$$

Lemma 5.12. Given a critical point $c_{0} \in \operatorname{Crit}_{\mathrm{p}}(f) \backslash \operatorname{Crit}_{\mathrm{per}}(f)$. Let $\left(K_{n}\left(c_{0}\right)\right.$, $\left.K_{n}^{-}\left(c_{0}\right)\right)_{n \geq 1}$ be the sequence of pairs of critical puzzle pieces constructed in Theorem 5.11. For $c \in\left[c_{0}\right] \backslash\left\{c_{0}\right\}$, let $K_{n}(c):=\mathcal{L}_{c}\left(K_{n}\left(c_{0}\right)\right)$. Then

(1) for every $c \in\left[c_{0}\right]$ and every $n \geq 1$, the restriction $\left.H\right|_{\partial K_{n}(c)}$ admits a qc extension inside $K_{n}(c)$ whose maximal dilatation is independent of $n$;

(2) for each $n \geq 1, \bigcup_{c \in\left[c_{0}\right]} K_{n}(c)$ is nice.

Proof. (1) We first prove that $\left.H\right|_{\partial K_{n}\left(c_{0}\right)}$ admits an $L^{\prime}$-qc extension inside $K_{n}\left(c_{0}\right)$ where $L^{\prime}$ is independent of $n$. This part is similar to the proof of Proposition 3.1 in 24 .

As $H$ preserves the degree information, the puzzle piece bounded by $H\left(\partial K_{n}\left(c_{0}\right)\right)$ (resp. $\left.H\left(\partial K_{n}^{-}\left(c_{0}\right)\right)\right)$ is a critical piece for $\tilde{f}$, denoted by $\tilde{K}_{n}\left(\tilde{c}_{0}\right)\left(\operatorname{resp} . H\left(\partial \tilde{K}_{n}^{-}\left(\tilde{c}_{0}\right)\right)\right)$, $\tilde{c}_{0} \in \operatorname{Crit}(\tilde{f})$.

Notice that $\left.H\right|_{\partial K_{1}\left(c_{0}\right)}$ has a qc extension on a neighborhood of $\partial K_{1}\left(c_{0}\right)$. It extends thus to an $L_{1}$-qc map $K_{1}\left(c_{0}\right) \rightarrow \tilde{K}_{1}\left(\tilde{c}_{0}\right)$, for some $L_{1} \geq 1$ (see e.g. [5], Lemma C.1).

In the construction of the sequence in Theorem 5.11, the operators $\Gamma, \mathcal{A}, \mathcal{B}$ are used. As they can be read off from the dynamical degree on the boundary of the puzzle pieces, and $H$ preserves this degree information, Theorem 5.11 is valid for the pair of sequences $\left(\tilde{K}_{n}\left(\tilde{c}_{0}\right), \tilde{K}_{n}^{-}\left(\tilde{c}_{0}\right)\right)_{n \geq 1}$ as well, with the same constant $S$, and probably a different $\tilde{\Delta}_{0}$ as a lower bound for $\bmod \left(\tilde{K}_{n}\left(\tilde{c}_{0}\right) \backslash \overline{\tilde{K}_{n}^{-}\left(\tilde{c}_{0}\right)}\right)$.

Recall that for each $i \geq 1$, the number $p_{i}$ denotes the integer such that $f^{p_{i}}\left(K_{i}\left(c_{0}\right)\right)$ $=K_{i-1}\left(c_{0}\right)$. We have $\tilde{f}^{p_{i}}\left(\tilde{K}_{i}\left(\tilde{c}_{0}\right)\right)=\tilde{K}_{i-1}\left(\tilde{c}_{0}\right)$, and both maps $f^{p_{i}}: K_{i}\left(c_{0}\right) \rightarrow$ $K_{i-1}\left(c_{0}\right)$ and $\tilde{f}^{p_{i}}: \tilde{K}_{i}\left(\tilde{c}_{0}\right) \rightarrow \tilde{K}_{i-1}\left(\tilde{c}_{0}\right)$ are proper holomorphic maps of degree at most $S$.

Now fix $n \geq 1$.

Set $v_{n}=c_{0}$, and then, for $i=n-1, n-2, \cdots, 1$, set consecutively $v_{i}=$ $f^{p_{i+1}+\ldots+p_{n}}\left(c_{0}\right)$.

Since $\left(K_{i}\left(c_{0}\right) \backslash K_{i}^{-}\left(c_{0}\right)\right) \cap \operatorname{orb}_{f}\left(\left[c_{0}\right]\right)=\emptyset$, all the critical values of $\left.f^{p_{i+1}}\right|_{K_{i+1}\left(c_{0}\right)}$, as well as $v_{i}$, are contained in $K_{i}^{-}\left(c_{0}\right), 1 \leq i \leq n-1$.

Let $\psi_{i}:\left(K_{i}\left(c_{0}\right), v_{i}\right) \rightarrow(\mathbb{D}, 0)$ be a bi-holomorphic uniformization, $i=1, \cdots, n$. For $i=2, \cdots, n$, let $g_{i}=\psi_{i-1} \circ f^{p_{i}} \circ \psi_{i}^{-1}$. These maps fix the point 0 , are proper holomorphic maps of degree at most $S$, with the critical values contained in $\psi_{i-1}\left(K_{i-1}^{-}\left(c_{0}\right)\right)$.

Let $\psi_{i}\left(K_{i}^{-}\left(c_{0}\right)\right)=\Omega_{i}$. Since $\left.\bmod \left(\mathbb{D} \backslash \overline{\Omega_{i}}\right)=\bmod \left(K_{i}\left(c_{0}\right)\right) \backslash \overline{K_{i}^{-}\left(c_{0}\right)}\right) \geq \Delta_{0}>0$ and $\Omega_{i} \ni \psi_{i}\left(v_{i}\right)=0,1 \leq i \leq n$, these domains are contained in some disk $\mathbb{D}_{s}$ with $s=s\left(\Delta_{0}\right)<1$. So the critical values of $g_{i}$ are contained in $\Omega_{i-1} \subset \mathbb{D}_{s}, 2 \leq i \leq n$.

The corresponding objects for $\tilde{f}$ will be marked with a tilde. The same assertions hold for $\tilde{g}_{i}$. Then all the maps $g_{i}$ and $\tilde{g}_{i}$ satisfy the assumptions of Lemma 5.9, 
with $d \leq S$, and $\rho=\max \{s, \tilde{s}\}$.

\begin{tabular}{|c|c|c|c|c|c|}
\hline$(\mathbb{D}, 0)$ & $\stackrel{\psi_{n}}{\longleftarrow}$ & $\left(K_{n}\left(c_{0}\right), v_{n}\right)$ & $\left(\tilde{K}_{n}\left(\tilde{c}_{0}\right), \tilde{v}_{n}\right)$ & $\stackrel{\tilde{\psi}_{n}}{\longrightarrow}$ & $(\mathbb{D}, 0)$ \\
\hline $\begin{array}{c}g_{n} \downarrow \\
(\mathbb{D}, 0)\end{array}$ & $\stackrel{\psi_{n-1}}{\longleftarrow}$ & $\begin{array}{c}\downarrow f^{p_{n}} \\
\left(K_{n-1}\left(c_{0}\right), v_{n-1}\right)\end{array}$ & $\begin{array}{c}\tilde{f}^{p_{n}} \downarrow \\
\left(\tilde{K}_{n-1}\left(\tilde{c}_{0}\right), \tilde{v}_{n-1}\right)\end{array}$ & $\stackrel{\tilde{\psi}_{n-1}}{\longrightarrow}$ & $\begin{array}{l}\downarrow \tilde{g}_{n} \\
(\mathbb{D}, 0)\end{array}$ \\
\hline$g_{n-1} \downarrow$ & & $\downarrow f^{p_{n-1}}$ & $\tilde{f}^{p_{n-1}} \downarrow$ & & $\downarrow \tilde{g}_{n-1}$ \\
\hline$\vdots$ & & $\vdots$ & $\vdots$ & & $\vdots$ \\
\hline$g_{3} \downarrow$ & & $\downarrow f^{p_{3}}$ & $\tilde{f}^{p_{3}} \downarrow$ & & $\downarrow \tilde{g}_{3}$ \\
\hline$(\mathbb{D}, 0)$ & $\stackrel{\psi_{2}}{\longleftarrow}$ & $\left(K_{2}\left(c_{0}\right), v_{2}\right)$ & $\left(\tilde{K}_{2}\left(\tilde{c}_{0}\right), \tilde{v}_{2}\right)$ & $\stackrel{\tilde{\psi}_{2}}{\longrightarrow}$ & $(\mathbb{D}, 0)$ \\
\hline$g_{2} \downarrow$ & & $\downarrow f^{p_{2}}$ & $\tilde{f}^{p_{2}} \downarrow$ & & $\downarrow \tilde{g}_{2}$ \\
\hline$(\mathbb{D}, 0)$ & $\stackrel{\psi_{1}}{\longleftarrow}$ & $\left(K_{1}\left(c_{0}\right), v_{1}\right)$ & $\left(\tilde{K}_{1}\left(\tilde{c}_{0}\right), \tilde{v}_{1}\right)$ & $\stackrel{\tilde{\psi}_{1}}{\longrightarrow}$ & $(\mathbb{D}, 0)$ \\
\hline
\end{tabular}

Note that each of $\psi_{i}, \tilde{\psi}_{i}$ extends to a homeomorphism from the closure of the puzzle piece to $\overline{\mathbb{D}}$.

Let us consider homeomorphisms $\eta_{i}: \mathbb{T} \rightarrow \mathbb{T}$ given by $\eta_{i}=\left.\tilde{\psi}_{i} \circ H\right|_{\partial K_{i}\left(c_{0}\right)} \circ \psi_{i}^{-1}$. They are equivariant with respect to the $g$-actions, i.e., $\eta_{i-1} \circ g_{i}=\tilde{g}_{i} \circ \eta_{i}$.

Due to the qc extension of $\left.H\right|_{\partial K_{1}\left(c_{0}\right)}$, we know that $\eta_{1}$ extends to an $L_{1}$-qc map $\mathbb{D} \rightarrow \mathbb{D}$. Then $\eta_{1}$ is an $L_{1}$-quasi-symmetric map. Fix some $r$ with $\rho<r<1$. We conclude that $\eta_{1}$ extends to an $L$-qc map $\xi_{1}: \mathbb{D} \rightarrow \mathbb{D}$ which is the identity on $\mathbb{D}_{r}$, where $L$ depends on $L_{1}, \rho$ and $r$.

Let $L_{0}=L_{0}(\rho, r, S)$ be as in Lemma 5.9, and let $L^{\prime}=\max \left\{L, L_{0}\right\}$. For $i=$ $2,3, \cdots, n$, apply consecutively Lemma 5.9 to the following left diagram (from bottom to top):

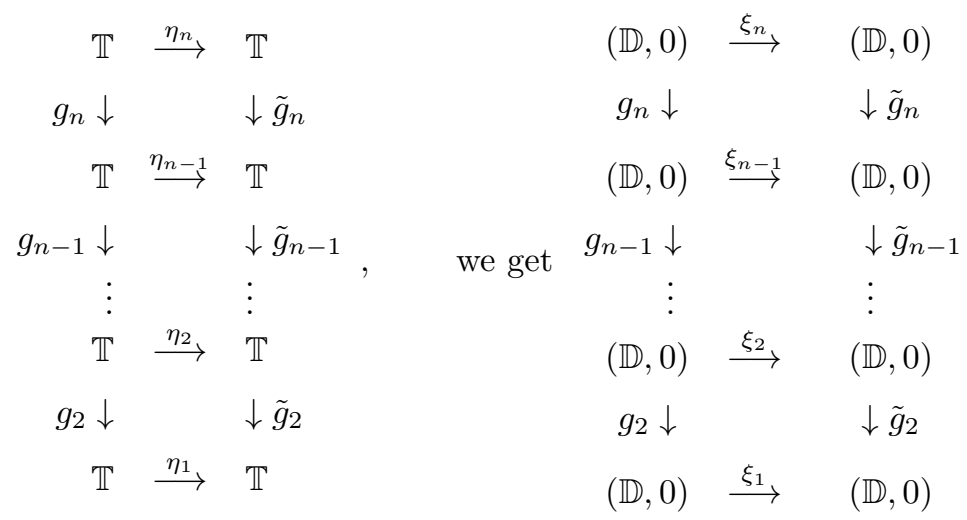

so that for $i=2, \ldots, n$, the map $\eta_{i}$ admits an $L^{\prime}$-qc extension $\xi_{i}: \mathbb{D} \rightarrow \mathbb{D}$ which is the identity on $\mathbb{D}_{r}$. The desired extension of $\left.H\right|_{\partial K_{n}\left(c_{0}\right)}$ inside $K_{n}\left(c_{0}\right)$ is now obtained by taking $\tilde{\psi}_{n}^{-1} \circ \xi_{n} \circ \psi_{n}$.

Now we show that for $c \in\left[c_{0}\right] \backslash\left\{c_{0}\right\}$, for each $n \geq 1,\left.H\right|_{\partial K_{n}(c)}$ admits an $\tilde{L}^{\prime}$-qc extension inside $K_{n}(c)$ with the constant $\tilde{L}^{\prime}$ independent of $n$.

Fix $n \geq 1$.

Let $f^{q_{n}}\left(K_{n}(c)\right)=K_{n}\left(c_{0}\right)$. Since $\left(K_{n}\left(c_{0}\right) \backslash K_{n}^{-}\left(c_{0}\right)\right) \cap \operatorname{orb}_{f}\left(\left[c_{0}\right]\right)=\emptyset$, all the critical values of $\left.f^{q_{n}}\right|_{K_{n}(c)}$ are contained in $K_{n}^{-}\left(c_{0}\right)$. 
Let $\varphi_{n}:\left(K_{n}\left(c_{0}\right), f^{q_{n}}(c)\right) \rightarrow(\mathbb{D}, 0)$ and $\lambda_{n}:\left(K_{n}(c), c\right) \rightarrow(\mathbb{D}, 0)$ be bi-holomorphic uniformizations. Set $\pi_{n}=\varphi_{n} \circ f^{q_{n}} \circ \lambda_{n}^{-1}$. This map fixes the point 0 , is a proper holomorphic map of degree at most $\delta^{b}$, with the critical values contained in $\varphi_{n}\left(K_{n}^{-}\left(c_{0}\right)\right)$.

Since

$$
\bmod \left(\mathbb{D} \backslash \overline{\varphi_{n}\left(K_{n}^{-}\left(c_{0}\right)\right)}\right)=\bmod \left(K_{n}\left(c_{0}\right) \backslash \overline{K_{n}^{-}\left(c_{0}\right)}\right) \geq \Delta_{0}>0
$$

and $\varphi_{n}\left(f^{q_{n}}(c)\right)=0$ belongs to $\varphi_{n}\left(K_{n}^{-}\left(c_{0}\right)\right)$, the set $\varphi_{n}\left(K_{n}^{-}\left(c_{0}\right)\right)$ is contained in the disk $\mathbb{D}_{s}$ (here $s$ is the same number as defined for the case of $c_{0}$ in this proof). So the critical values of $\pi_{n}$ are contained in $\varphi_{n}\left(K_{n}^{-}\left(c_{0}\right)\right) \subset \mathbb{D}_{s}$ :

$$
\begin{array}{cccccc}
(\mathbb{D}, 0) & \stackrel{\lambda_{n}}{\longleftarrow} & \left(K_{n}(c), c\right) & \left(\tilde{K}_{n}(\tilde{c}), \tilde{c}\right) & \stackrel{\tilde{\lambda}_{n}}{\longrightarrow} & (\mathbb{D}, 0) \\
\pi_{n} \downarrow & & \downarrow f^{q_{n}} & \tilde{f}^{q_{n}} \downarrow & & \downarrow \tilde{\pi}_{n} \\
(\mathbb{D}, 0) & \stackrel{\varphi_{n}}{\longleftarrow} & \left(K_{n}\left(c_{0}\right), f^{q_{n}}(c)\right) & \left(\tilde{K}_{n}\left(\tilde{c}_{0}\right), \tilde{f}^{q_{n}}(\tilde{c})\right) & \stackrel{\tilde{\varphi}_{n}}{\longrightarrow} & (\mathbb{D}, 0)
\end{array}
$$

Let $\tilde{K}_{n}(\tilde{c}), \tilde{c} \in \operatorname{Crit}(\tilde{f})$ be the puzzle piece bounded by $H\left(\partial K_{n}(c)\right)$. The corresponding objects for $\tilde{f}$ will be marked with a tilde. The same assertions hold for $\tilde{\pi}_{n}$. Then both maps $\pi_{n}$ and $\tilde{\pi}_{n}$ satisfy the assumptions of Lemma 5.9, with $d \leq \delta^{b}$, and $\rho=\max \{s, \tilde{s}\}$.

Note that each of $\varphi_{n}, \tilde{\varphi}_{n}, \lambda_{n}, \tilde{\lambda}_{n}$ extends to a homeomorphism from the closure of the puzzle piece to $\overline{\mathbb{D}}$.

Let us consider homeomorphisms $\alpha_{n}: \mathbb{T} \rightarrow \mathbb{T}$ and $\beta_{n}: \mathbb{T} \rightarrow \mathbb{T}$ given by $\alpha_{n}=$ $\left.\tilde{\varphi}_{n} \circ H\right|_{\partial K_{n}\left(c_{0}\right)} \circ \varphi_{n}^{-1}$ and $\beta_{n}=\left.\tilde{\lambda}_{n} \circ H\right|_{\partial K_{n}(c)} \circ \lambda_{n}^{-1}$. Then $\beta_{n} \circ \pi_{n}=\pi_{n} \circ \alpha_{n}$.

Due to the $L^{\prime}$-qc extension of $\left.H\right|_{\partial K_{n}\left(c_{0}\right)}$, we know that $\alpha_{n}$ extends to an $L^{\prime}$-qc map $\mathbb{D} \rightarrow \mathbb{D}$. We still fix the number $r$ with $\rho<r<1$. We can extend $\alpha_{n}$ to be an $\tilde{L}$-qc map $\mu_{n}: \mathbb{D} \rightarrow \mathbb{D}$ which is the identity on $\mathbb{D}_{r}$, where $\tilde{L}$ depends on $L^{\prime}, \rho$ and $r$.

Let $\tilde{L}_{0}=\tilde{L}_{0}\left(\rho, r, \delta^{b}\right)$ be as in Lemma [5.9, and let $\tilde{L}^{\prime}=\max \left\{\tilde{L}, \tilde{L}_{0}\right\}$. We apply Lemma 5.9 to the following left diagram:

$$
\begin{aligned}
& \mathbb{T} \stackrel{\beta_{n}}{\longrightarrow} \mathbb{T} \quad(\mathbb{D}, 0) \stackrel{\nu_{n}}{\longrightarrow}(\mathbb{D}, 0) \\
& \pi_{n} \downarrow \quad \downarrow \tilde{\pi}_{n}, \quad \text { we get } \pi_{n} \downarrow \quad \downarrow \tilde{\pi}_{n} \\
& \mathbb{T} \stackrel{\alpha_{n}}{\longrightarrow} \mathbb{T} \quad(\mathbb{D}, 0) \stackrel{\mu_{n}}{\longrightarrow}(\mathbb{D}, 0)
\end{aligned}
$$

so that the map $\beta_{n}$ admits an $\tilde{L}^{\prime}$-qc extension $\nu_{n}: \mathbb{D} \rightarrow \mathbb{D}$ which is the identity on $\mathbb{D}_{r}$. The desired extension of $\left.H\right|_{\partial K_{n}(c)}$ inside $K_{n}(c)$ is obtained by taking $\tilde{\lambda}_{n}^{-1} \circ \nu_{n} \circ$ $\lambda_{n}$.

(2) The fact that the set $\bigcup_{c \in\left[c_{0}\right]} K_{n}(c)$ is nice follows directly from Lemma 5.7

Lemma 5.13. Given $c_{0} \in \operatorname{Crit}_{\mathrm{n}}(f) \cup \operatorname{Crit}_{\mathrm{r}}(f)$. Then there exist a puzzle piece $P$ of depth $n_{0}$, a topological disk $T \subset \subset P$, and for each $c \in \operatorname{Crit}(f)$ with $c=c_{0}$ or $c \rightarrow c_{0}$, there is a nested sequence of puzzle pieces containing $c$, denoted by $\left\{K_{n}(c)\right\}_{n \geq 1}$, satisfying the following properties.

(1) Every $K_{n}(c)$ is a pullback of $P$, that is, $f^{s_{n}}\left(K_{n}(c)\right)=P$ for some $s_{n} \geq 1$. Furthermore, $\operatorname{deg}\left(f^{s_{n}}: K_{n}(c) \rightarrow P\right) \leq \delta^{b+1}$ and all critical values of the map $f^{s_{n}}: K_{n}(c) \rightarrow P$ are contained in $T$.

(2) $\left.H\right|_{\partial K_{n}(c)}$ admits a qc extension inside $K_{n}(c)$ with a maximal dilatation independent of $n$.

(3) For every $n \geq 1, \bigcup_{c \in\left[c_{0}\right]} K_{n}(c)$ is a nice set. 
Proof. (1) Suppose $c_{0} \in \operatorname{Crit}_{\mathrm{n}}(f)$ and then $\left[c_{0}\right]=\left\{c_{0}\right\}$. In $\mathcal{T}\left(c_{0}\right) \backslash\{0$-th column $\}$, every vertex is noncritical. So for each $n \geq 1$,

$$
\operatorname{deg}\left(f^{n}: P_{n}\left(c_{0}\right) \rightarrow P_{0}\left(f^{n}\left(c_{0}\right)\right)\right)=\operatorname{deg}_{c_{0}}(f) \leq \delta .
$$

Since there are finitely many puzzle pieces of the same depth, we can take a subsequence $\left\{u_{n}\right\}_{n \geq 1}$ such that $f^{u_{n}}\left(P_{u_{n}}\left(c_{0}\right)\right)=P$ for some fixed puzzle piece $P$ of depth 0 .

Given $c \rightarrow c_{0}, c \in \operatorname{Crit}(f)$, let $f^{v_{n}}\left(\mathcal{L}_{c}\left(P_{u_{n}}\left(c_{0}\right)\right)=P_{u_{n}}\left(c_{0}\right)\right.$. Then

$$
\begin{aligned}
& \operatorname{deg}\left(f^{v_{n}+u_{n}}: \mathcal{L}_{c}\left(P_{u_{n}}\left(c_{0}\right)\right) \rightarrow P\right) \\
= & \operatorname{deg}\left(f^{v_{n}}: \mathcal{L}_{c}\left(P_{u_{n}}\left(c_{0}\right)\right) \rightarrow P_{u_{n}}\left(c_{0}\right)\right) \cdot \operatorname{deg}\left(f^{u_{n}}: P_{u_{n}}\left(c_{0}\right) \rightarrow P\right) \\
\leq & \delta^{b} \cdot \delta .
\end{aligned}
$$

For $c_{0} \in \operatorname{Crit}_{\mathrm{n}}(f)$, we set $K_{n}\left(c_{0}\right)=P_{u_{n}}\left(c_{0}\right)$ and $s_{n}=u_{n}$. For $c \rightarrow c_{0}, c \in \operatorname{Crit}(f)$, set $K_{n}(c)=\mathcal{L}_{c}\left(P_{u_{n}}\left(c_{0}\right)\right)$ and $s_{n}=v_{n}+u_{n}$.

Now suppose $c_{0} \in \operatorname{Crit}_{\mathrm{r}}(f)$ and $c \rightarrow c_{0}, c \in \operatorname{Crit}(f)$. Since $\mathcal{T}\left(c_{0}\right)$ is reluctantly recurrent, there exist an integer $n_{0} \geq 0, c_{1}, c_{2} \in\left[c_{0}\right]$ and infinitely many integers $l_{n} \geq 1$ such that $\left\{P_{n_{0}+l_{n}}\left(c_{2}\right)\right\}_{n \geq 1}$ are children of $P_{n_{0}}\left(c_{1}\right)$ and then $\operatorname{deg}\left(f^{l_{n}}\right.$ : $\left.P_{n_{0}+l_{n}}\left(c_{2}\right) \rightarrow P_{n_{0}}\left(c_{1}\right)\right)=\operatorname{deg}_{c_{2}}(f) \leq \delta$. Suppose $f^{k_{n}}\left(\mathcal{L}_{c}\left(P_{n_{0}+l_{n}}\left(c_{2}\right)\right)\right)=P_{n_{0}+l_{n}}\left(c_{2}\right)$ for $k_{n} \geq 1$. Then

$$
\begin{aligned}
& \operatorname{deg}\left(f^{l_{n}+k_{n}}: P_{n_{0}+l_{n}+k_{n}}(c) \rightarrow P_{n_{0}}\left(c_{1}\right)\right) \\
= & \operatorname{deg}\left(f^{k_{n}}: \mathcal{L}_{c}\left(P_{n_{0}+l_{n}}\left(c_{2}\right)\right) \rightarrow P_{n_{0}+l_{n}}\left(c_{2}\right)\right) \cdot \operatorname{deg}\left(f^{l_{n}}: P_{n_{0}+l_{n}}\left(c_{2}\right) \rightarrow P_{n_{0}}\left(c_{1}\right)\right) \\
\leq & \delta^{b} \cdot \delta
\end{aligned}
$$

Take a strictly increasing subsequence of $l_{n}$, which is still denoted by $l_{n}$, such that $\left\{P_{n_{0}+l_{n}}\left(c_{2}\right)\right\}_{n \geq 1}$ is a nested sequence of puzzle pieces containing $c_{2}$ and then $\left\{P_{n_{0}+l_{n}+k_{n}}(c)\right\}_{n \geq 1}$ is a nested sequence of puzzle pieces containing $c$. Set $P=$ $P_{n_{0}}\left(c_{1}\right)$. For $c=c_{2}$, set $s_{n}=l_{n}$ and $K_{n}(c)=P_{n_{0}+l_{n}}(c)$; for $c \neq c_{2}$, set $s_{n}=k_{n}+l_{n}$ and $K_{n}(c)=P_{n_{0}+k_{n}+l_{n}}(c)$.

Now fix $c_{0} \in \operatorname{Crit}_{\mathrm{n}}(f) \cup \mathrm{Crit}_{\mathrm{r}}(f)$. Take a topological disk $T \subset \subset P$ such that $T$ contains all the puzzle pieces of depth $n_{0}+1$. For each $c=c_{0}$ or $c \rightarrow c_{0}, c \in \operatorname{Crit}(f)$, and each $n \geq 1$, all critical values of $\left.f^{s_{n}}\right|_{K_{n}(c)}$ are contained in the union of puzzle pieces of depth $n_{0}+1$ because $\operatorname{Crit}(f) \subset \mathbf{K}_{f}$ and $f\left(\mathbf{K}_{f}\right)=\mathbf{K}_{f}$. Consequently, the set $T$ contains all the critical values of the map $\left.f^{s_{n}}\right|_{K_{n}(c)}$.

(2) We will use Lemma 5.9 to construct the qc extension.

Fix $c=c_{0}$ or $c \rightarrow c_{0}$ and $n \geq 1$.

Let $\tilde{P}$ and $\tilde{K}_{n}(\tilde{c})$ be the puzzle pieces for $\tilde{f}$ bounded by $H(\partial P)$ and $H\left(\partial K_{n}(c)\right)$ respectively. Since $H$ preserves the degree information, for the map $\tilde{f}$, we also have the similar statement as for $f$ in $(1)$, more precisely, $\tilde{f}^{s_{n}}\left(\tilde{K}_{n}(\tilde{c})\right)=\tilde{P}, \operatorname{deg}\left(\tilde{f}^{s_{n}}\right.$ : $\left.\tilde{K}_{n}(\tilde{c}) \rightarrow \tilde{P}\right) \leq \delta^{b+1}$, and all critical values of the map $\tilde{f}^{s_{n}}: \tilde{K}_{n}(\tilde{c}) \rightarrow \tilde{P}$ are contained in $\tilde{T}$, where $\tilde{T}$ is a topological disk in $\tilde{P}$ containing all puzzle pieces for $\tilde{f}$ of depth $n_{0}+1$ in $\tilde{P}$.

Let $\bmod (P \backslash \bar{T})=\Delta_{1}$ and $\bmod (\tilde{P} \backslash \overline{\tilde{T}})=\tilde{\Delta}_{1}$.

Let $\iota_{n}:\left(P, f^{s_{n}}(c)\right) \rightarrow(\mathbb{D}, 0)$ and $\theta_{n}:\left(K_{n}(c), c\right) \rightarrow(\mathbb{D}, 0)$ be bi-holomorphic uniformizations. Let $h_{n}=\iota_{n} \circ f^{s_{n}} \circ \theta_{n}^{-1}$. Then $h_{n}$ fixes the point 0 , is a proper holomorphic map of degree at most $\delta^{b+1}$, with all the critical values contained in $\iota_{n}(T)$. 
Since $\bmod \left(\mathbb{D} \backslash \overline{\iota_{n}(T)}\right)=\bmod (P \backslash \bar{T})=\Delta_{1}>0$ and $\iota_{n}(T) \ni \iota_{n}\left(f^{s_{n}}(c)\right)=0$, we have $\iota_{n}(T) \subset \mathbb{D}_{t}$ with $t=t\left(\Delta_{1}\right)<1$. So the critical values of $h_{n}$ are contained in $\iota_{n}(T) \subset \mathbb{D}_{t}$.

The corresponding objects for $\tilde{f}$ will be marked with a tilde. The same assertions hold for $\tilde{h}_{n}$. Then the maps $h_{n}$ and $\tilde{h}_{n}$ satisfy the assumptions of Lemma 5.9, with $d \leq \delta^{b+1}$, and $\rho=\max \{t, \tilde{t}\}$.

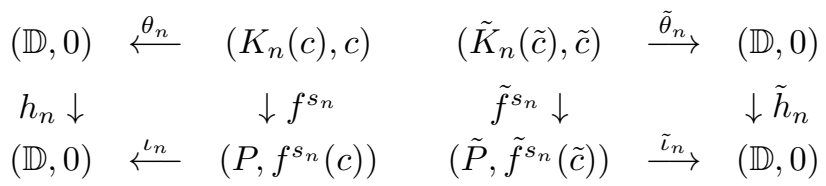

Note that each of $\iota_{n}, \tilde{\iota}_{n}, \theta_{n}, \tilde{\theta}_{n}$ extends to a homeomorphism from the closure of the puzzle piece to $\overline{\mathbb{D}}$.

Let us consider homeomorphisms $\kappa_{n}: \mathbb{T} \rightarrow \mathbb{T}$ and $\sigma_{n}: \mathbb{T} \rightarrow \mathbb{T}$ given by $\kappa_{n}=$ $\left.\tilde{\iota}_{n} \circ H\right|_{\partial P} \circ \iota_{n}^{-1}$ and $\sigma_{n}=\left.\tilde{\theta}_{n} \circ H\right|_{\partial K_{n}(c)} \circ \theta_{n}^{-1}$, respectively. Then $\kappa_{n} \circ h_{n}=\tilde{h}_{n} \circ \sigma_{n}$.

Notice that $\left.H\right|_{\partial P}$ has a $K_{1}$-qc extension in $P$ for some $K_{1} \geq 1$. The number $K_{1}$ is independent of $n$ because the choice of $P$ does not depend on $n$. Fix some $r$ with $\rho<r<1$. We conclude that $\kappa_{n}$ extends to a $K$-qc map $\omega_{n}: \mathbb{D} \rightarrow \mathbb{D}$ which is the identity on $\mathbb{D}_{r}$, where $K$ depends on $K_{1}, \rho$ and $r$.

Let $K_{0}=K_{0}\left(\rho, r, \delta^{b+1}\right)$ be as in Lemma 5.9 and let $K^{\prime}=\max \left\{K, K_{0}\right\}$. Apply Lemma 5.9 to the following left diagram:

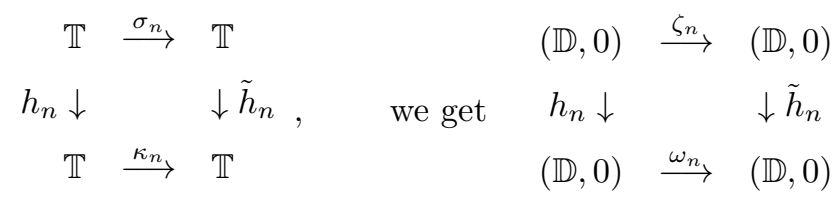

so that the map $\sigma_{n}$ admits a $K^{\prime}$-qc extension $\zeta_{n}: \mathbb{D} \rightarrow \mathbb{D}$ which is the identity on $\mathbb{D}_{r}$. The desired extension of $\left.H\right|_{\partial K_{n}(c)}$ inside $K_{n}(c)$ is now obtained by taking $\tilde{\theta}_{n}^{-1} \circ \zeta_{n} \circ \theta_{n}$.

(3) Fix $n \geq 1$.

For $c_{0} \in \operatorname{Crit}_{\mathrm{n}}(f)$, since $\left[c_{0}\right]=\left\{c_{0}\right\}$, we know that $\bigcup_{c \in\left[c_{0}\right]} K_{n}(c)=K_{n}\left(c_{0}\right)$ and obviously $K_{n}\left(c_{0}\right)$ is nice.

For the case $c_{0} \in \mathrm{Crit}_{\mathrm{r}}(f)$, we apply Lemma 5.7 to

$$
\bigcup_{c \in\left[c_{0}\right]} K_{n}(c)=\bigcup_{c \in\left[c_{0}\right] \backslash\left\{c_{2}\right\}} \mathcal{L}_{c}\left(P_{n_{0}+l_{n}}\left(c_{2}\right)\right) \cup P_{n_{0}+l_{n}}\left(c_{2}\right)
$$

and easily get the conclusion.

Lemma 5.14. Suppose $c_{0} \in \operatorname{Crit}_{\mathrm{e}}(f)$. Then

(1) there is a nested sequence of puzzle pieces containing $c_{0}$, denoted by $\left\{K_{n}\left(c_{0}\right)\right\}_{n \geq 1}$, such that for each $n \geq 1,\left.H\right|_{\partial K_{n}\left(c_{0}\right)}$ admits a qc extension inside $K_{n}\left(c_{0}\right)$ with a maximal dilatation independent of $n$,

(2) for every $n \geq 1, \bigcup_{c \in\left[c_{0}\right]} K_{n}(c)$ is a nice set.

Proof. Suppose $c_{0} \in \mathrm{Crit}_{\mathrm{e}}(f)$. In the following, we will discuss the three cases indicated in Lemma 5.6 (ii).

Case 1. Forw $\left(c_{0}\right) \cap\left(\operatorname{Crit}_{\mathrm{n}}(f) \cup \operatorname{Crit}_{\mathrm{r}}(f)\right) \neq \emptyset$. By using Lemma 5.13, we can get a nested sequence of puzzle pieces containing $c_{0}$, denoted by $\left\{K_{n}\left(c_{0}\right)\right\}_{n \geq 1}$, 
and $\left.H\right|_{\partial K_{n}(c)}$ admits a qc extension inside $K_{n}\left(c_{0}\right)$ whose maximal dilatation is independent of $n$.

We divide Case $2\left(\operatorname{Forw}\left(c_{0}\right) \subset \operatorname{Crit}_{\mathrm{p}}(f)\right)$ into two subcases.

Subcase 1. There is a critical point $c_{1} \in \operatorname{Forw}\left(c_{0}\right) \cap\left(\operatorname{Crit}_{\mathrm{p}}(f) \backslash \operatorname{Crit}_{\text {per }}(f)\right)$. Let $\left(K_{n}\left(c_{1}\right), K_{n}^{-}\left(c_{1}\right)\right)_{n \geq 1}$ be the sequence of pairs of critical puzzle pieces constructed in Theorem 5.11.

For $n \geq 1$, set

$$
K_{n}^{-}\left(c_{0}\right)=\mathcal{L}_{c_{0}}\left(K_{n}^{-}\left(c_{1}\right)\right), f^{r_{n}}\left(K_{n}^{-}\left(c_{0}\right)\right)=K_{n}^{-}\left(c_{1}\right)
$$

and

$$
K_{n}\left(c_{0}\right)=\operatorname{Comp}_{c_{0}}\left(f^{-r_{n}}\left(K_{n}\left(c_{1}\right)\right)\right) .
$$

Clearly, $\left(K_{n}\left(c_{0}\right) \backslash K_{n}^{-}\left(c_{0}\right)\right) \cap \operatorname{Crit}(f)=\emptyset$. Since $\left(K_{n}\left(c_{1}\right) \backslash K_{n}^{-}\left(c_{1}\right)\right) \cap \operatorname{orb}_{f}\left(\left[c_{1}\right]\right)=$ $\emptyset$ and Forw $\left(c_{1}\right)=\left[c_{1}\right]$, by Lemma 5.8, we conclude that for all $1 \leq i<r_{n}$, $\left(f^{i}\left(K_{n}\left(c_{0}\right)\right) \backslash f^{i}\left(K_{n}^{-}\left(c_{0}\right)\right)\right) \cap \operatorname{Forw}\left(c_{1}\right)=\emptyset$.

We claim that for every $n \geq 1$ and every $1 \leq i<r_{n}$,

$$
\left(f^{i}\left(K_{n}\left(c_{0}\right)\right) \backslash f^{i}\left(K_{n}^{-}\left(c_{0}\right)\right)\right) \cap\left(\operatorname{Crit}(f) \backslash \operatorname{Forw}\left(c_{1}\right)\right)=\emptyset .
$$

If not, there is some $n$ such that

$$
\left\{f\left(K_{n}\left(c_{0}\right)\right) \backslash f\left(K_{n}^{-}\left(c_{0}\right)\right), \cdots, f^{r_{n}-1}\left(K_{n}\left(c_{0}\right)\right) \backslash f^{r_{n}-1}\left(K_{n}^{-}\left(c_{0}\right)\right)\right\}
$$

meets some critical point, say $c_{2} \in \operatorname{Crit}(f) \backslash \operatorname{Forw}\left(c_{1}\right)$. See Figure 7

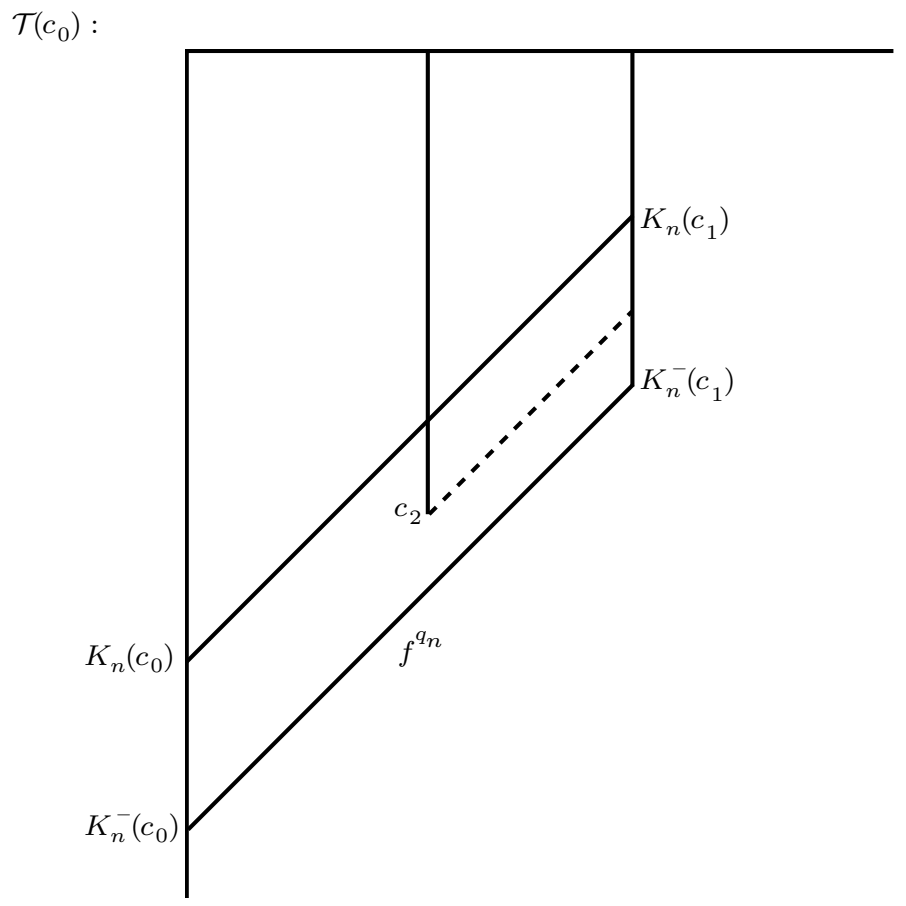

FiguRE 7

Then $c_{0} \rightarrow c_{2} \rightarrow c_{1}$. Since Forw $\left(c_{0}\right) \subset \operatorname{Crit}_{\mathrm{p}}(f)$, we have $c_{2} \in \operatorname{Crit}_{\mathrm{p}}(f)$ and then Forw $\left(c_{2}\right)=\left[c_{2}\right]$. So $c_{1} \rightarrow c_{2}$. It contradicts $c_{2} \notin \operatorname{Forw}\left(c_{1}\right)$. 
Hence for every $n \geq 1$ and every $0 \leq i<r_{n}$,

$$
\left(f^{i}\left(K_{n}\left(c_{0}\right)\right) \backslash f^{i}\left(K_{n}^{-}\left(c_{0}\right)\right)\right) \cap \operatorname{Crit}(f)=\emptyset .
$$

From the equation (5.2) and $K_{n}^{-}\left(c_{0}\right)=\mathcal{L}_{c_{0}}\left(K_{n}^{-}\left(c_{1}\right)\right)$, we conclude that for $n \geq 1$,

$$
\operatorname{deg}\left(f^{r_{n}}: K_{n}\left(c_{0}\right) \rightarrow K_{n}\left(c_{1}\right)\right) \leq \delta^{b} .
$$

Again by the equation (5.2), we know that all critical values of the map $f^{r_{n}}$ : $K_{n}\left(c_{0}\right) \rightarrow K_{n}\left(c_{1}\right)$ are contained in $K_{n}^{-}\left(c_{1}\right)$.

Using a similar method as in the proof of Lemma 5.12 (1), we could obtain a qc extension of $\left.H\right|_{\partial K_{n}\left(c_{0}\right)}$ inside $K_{n}\left(c_{0}\right)$ whose maximal dilatation is independent of $n$. We omit the details here.

Subcase 2. Suppose Forw $\left(\mathrm{c}_{0}\right) \subset \operatorname{Crit}_{\text {per }}(f)$.

If $f^{l}\left(\mathbf{K}_{f}\left(c_{0}\right)\right)$ is periodic for some $l \geq 1$, then there is some critical periodic component in the periodic cycle of $f^{l}\left(\mathbf{K}_{f}\left(c_{0}\right)\right)$. By the assumption $(* *)$ in Proposition 5.3, there is an integer $N_{c_{0}}$ such that $\left.H\right|_{\partial P_{N_{c_{0}}+n}\left(c_{0}\right)}$ has an $M_{c_{0}}$-qc extension, where $M_{c_{0}}$ is independent of $n$. Set $K_{n}\left(c_{0}\right):=P_{N_{c_{0}}+n}\left(c_{0}\right)$. We are done.

Now we suppose that $\mathbf{K}_{f}\left(c_{0}\right)$ is wandering. For each $\hat{c} \in \operatorname{Forw}\left(\mathrm{c}_{0}\right)$, by the condition of Proposition [5.3. there are a constant $M_{\hat{c}}$ and an integer $N_{\hat{c}}$ such that the map $\left.H\right|_{\partial P_{n}(\hat{c})}$ extends to an $M_{\hat{c}-q c}$ extension inside $P_{n}(\hat{c})$ for all $n \geq N_{\hat{c}}$. Set $N:=\max \left\{N_{\hat{c}}, \hat{c} \in \operatorname{Forw}\left(\mathrm{c}_{0}\right)\right\}$.

Claim 1. There are a point $c_{1} \in$ Forw $\left(c_{0}\right)$, a topological disk $T_{1} \subset \subset P_{N}\left(c_{1}\right)$ and a nested sequence of puzzle pieces containing $c_{0}$, denoted by $\left\{K_{n}\left(c_{0}\right)\right\}_{n \geq 1}$, satisfying the following conditions: for every $n \geq 1$, there exists an integer $w_{n} \geq 1$ such that

(i) $f^{w_{n}}\left(K_{n}\left(c_{0}\right)\right)=P_{N}\left(c_{1}\right)$,

(ii) $\operatorname{deg}\left(f^{w_{n}}: K_{n}\left(c_{0}\right) \rightarrow P_{N}\left(c_{1}\right)\right) \leq \delta^{b}$,

(iii) all critical values of the map $\left.f^{w_{n}}\right|_{K_{n}\left(c_{0}\right)}$ are contained in the set $T_{1}$.

Proof. Suppose $c \in \operatorname{Forw}\left(c_{0}\right)$. Refer to Figure 8 for the proof.

Since $\mathbf{K}_{f}\left(c_{0}\right)$ is wandering, in $\mathcal{T}\left(c_{0}\right)$, there are infinitely many vertices $\left\{\left(-\left(N+m_{n}\right), k_{n}\right)\right\}_{n \geq 1}$ such that $\left(-\left(N+m_{n}\right), k_{n}\right)$ is the first vertex being $c$ on the $m_{n}$-th row, $\left(-\left(N+m_{n}+1\right), k_{n}\right)$ is not critical and $\lim _{n \rightarrow \infty} m_{n}=\infty$. Then

$$
f^{k_{n}}\left(\mathcal{L}_{c_{0}}\left(P_{N+m_{n}}(c)\right)\right)=P_{N+m_{n}}(c)
$$

and $\operatorname{deg}\left(f^{k_{n}}: P_{N+m_{n}+k_{n}}\left(c_{0}\right) \rightarrow P_{N+m_{n}}(c)\right) \leq \delta^{b}$.

Let $p$ be the period of $\mathbf{K}_{f}(c)$. Then in $\mathcal{T}(c)$, for every $0<j<p$, either $\left(-\left(N+m_{n}-j\right), j\right)$ is not critical or $\left(-\left(N+m_{n}+1-j\right), j\right)$ is critical. Using (Rule 3) several times, we conclude that in $\mathcal{T}\left(c_{0}\right)$, there are no critical vertices on the diagonal starting from the vertex at $\left(-\left(N+m_{n}+1\right), k_{n}\right)$ to the vertex at $\left(-(N+1), m_{n}+k_{n}\right)$. From the vertex $\left(-N, m_{n}+k_{n}\right)$, march horizontally $l_{n} \geq 1$ steps until the first hit of some $c_{1}(n)$ vertex for some $c_{1}(n) \in \operatorname{Forw}\left(c_{0}\right)$. Then in $\mathcal{T}\left(c_{0}\right)$, there is no critical vertex on the diagonal starting from the vertex $\left(-\left(N+m_{n}+k_{n}+l_{n}-1\right), 1\right)$ to the vertex $\left(-(N+1), m_{n}+k_{n}+l_{n}-1\right)$. Therefore,

$$
\operatorname{deg}\left(f^{m_{n}+k_{n}+l_{n}}: P_{N+m_{n}+k_{n}+l_{n}}\left(c_{0}\right) \rightarrow P_{N}\left(c_{1}(n)\right)\right) \leq \delta^{b} .
$$

Since $c_{1}(n)$ belongs to the finite set Forw $\left(\mathrm{c}_{0}\right)$ and $m_{n} \rightarrow \infty$ as $n \rightarrow \infty$, we could find a subsequence of $n$, say itself, such that $\left\{P_{N+m_{n}+k_{n}+l_{n}}\left(c_{0}\right)\right\}_{n \geq 1}$ form a nested sequence and $c_{1}(n) \equiv c_{1}$. Set $w_{n}:=m_{n}+k_{n}+l_{n}$ and $K_{n}\left(c_{0}\right):=\bar{P}_{N+w_{n}}\left(c_{0}\right)$. 


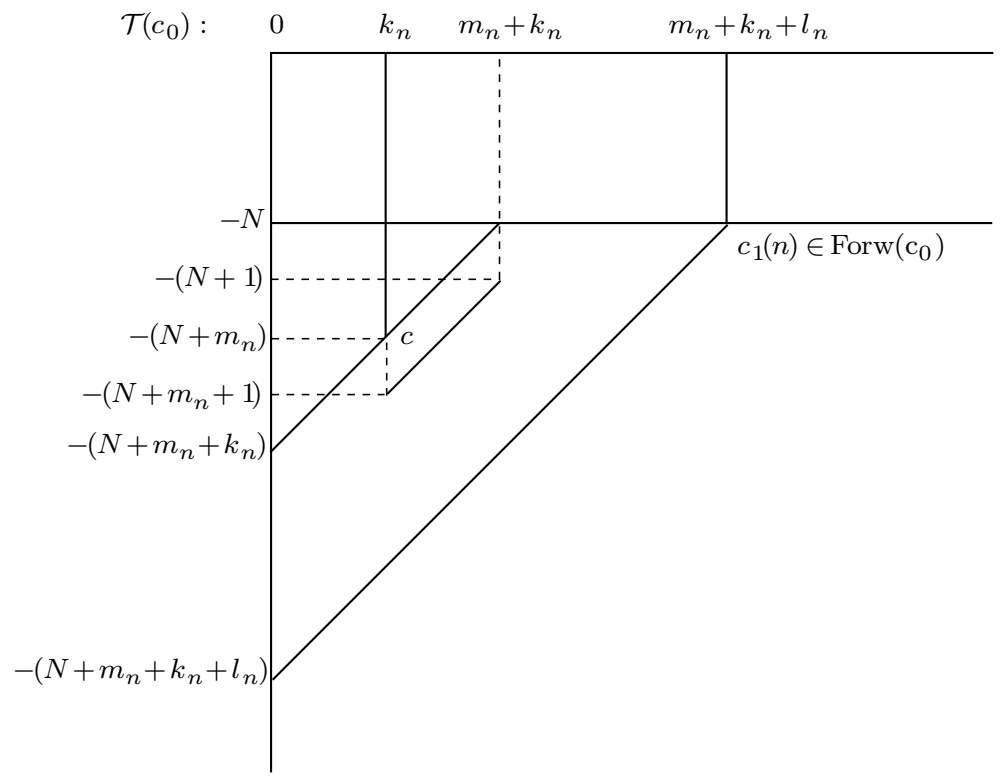

Figure 8

Similarly to the proof of Lemma 5.13 (1), one takes a topological disk $T_{1} \subset \subset$ $P_{N}\left(c_{1}\right)$ such that all the puzzle pieces of depth $N+1$ are contained in $T_{1}$ and particularly, all of the critical values of $\left.f^{w_{n}}\right|_{K_{n}\left(c_{0}\right)}$ are contained in $T_{1}$.

Using a similar method as in the proof of Lemma 5.13 (2), we could show that $\left.H\right|_{\partial K_{n}\left(c_{0}\right)}$ admits a qc extension inside $K_{n}\left(c_{0}\right)$ whose maximal dilatation is independent of $n$.

In Case 3, we will first draw the similar conclusion to Lemma 5.13 (1).

Take arbitrarily a point $c \in \operatorname{Crit}_{\mathrm{e}}(f) \cap \operatorname{Forw}\left(c_{0}\right)$. In $T\left(c_{0}\right)$, let $\left\{\left(0, t_{n}\right)\right\}_{n \geq 1}$ be all the $c$-vertices on the 0 -th row with $1 \leq t_{1}<t_{2}<\cdots$.

Since $c_{0} \in \operatorname{Crit}_{\mathrm{e}}(f)$ and then $c_{0} \not \rightarrow c_{0}$, by the assumption $(* * *)$, the $c_{0}$-vertex will not appear in $\mathcal{T}\left(c_{0}\right) \backslash\{0$-th column $\}$. In particular, for each $n \geq 1$, there are no $c_{0}$-vertices on the diagonal starting from the vertex $\left(-\left(t_{n}-1\right), 1\right)$ and ending at the vertex $\left(-1, t_{n}-1\right)$. Denote that diagonal by $J_{n}$. Since $c \not \rightarrow c$, by the assumption $(* * *)$, there are no $c$-vertices on the diagonal $J_{n}$.

We claim that for every $n \geq 1$, the diagonal $J_{n}$ meets every point in $\operatorname{Crit}(f) \backslash\left\{c_{0}, c\right\}$ at most once. In fact, if not, then there is some $n^{\prime}$ and some $c^{\prime} \in \operatorname{Crit}(f)$ such that the diagonal $J_{n^{\prime}}$ meets $c^{\prime}$ at least twice. By the assumption $(* * *)$, we can conclude that $c_{0} \rightarrow c^{\prime} \rightarrow c^{\prime} \rightarrow c$; see Figure 9. By the condition of Case 3 and $c^{\prime} \rightarrow c^{\prime}$, we know that $c^{\prime} \in \operatorname{Crit}_{\mathrm{p}}(f)$ and then Forw $\left(c^{\prime}\right)=\left[c^{\prime}\right]$ by Lemma 5.5. Thus $c \rightarrow c^{\prime}$ and then $c \rightarrow c$. This contradicts $c \in \operatorname{Crit}_{\mathrm{e}}(f)$.

By the argument above, one easily finds that $\left\{P_{t_{n}}\left(c_{0}\right)\right\}_{n \geq 1}$ is a nested sequence of puzzle pieces containing $c_{0}$ and $\operatorname{deg}\left(f^{t_{n}}: P_{t_{n}}\left(c_{0}\right) \rightarrow P_{0}(c)\right) \leq \delta^{b-1}$. Let $T_{2}$ be a topological disk compactly contained in $P_{0}(c)$ such that $T_{2}$ contains all the puzzle pieces of depth 1 in $P_{0}(c)$. Notice that all the critical values are contained in the union of all the puzzle pieces of depth 1 in $P_{0}(c)$, so they are also contained in $T_{2}$. 


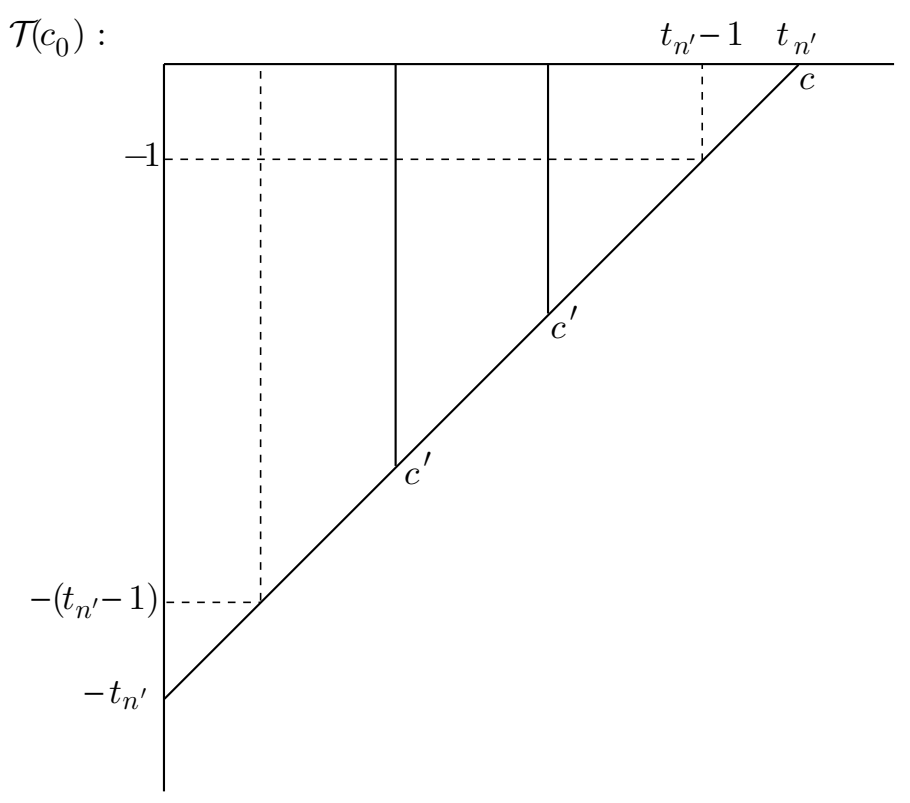

Figure 9

Set $K_{n}\left(c_{0}\right):=P_{t_{n}}\left(c_{0}\right)$ and then we get similar objects as in Lemma $5.13(1)$. Using a similar method in the proof of Lemma $5.13(2)$, we could show that $\left.H\right|_{\partial K_{n}\left(c_{0}\right)}$ admits a qc extension inside $K_{n}\left(c_{0}\right)$ whose maximal dilatation is independent of $n$.

(2) Since $c_{0} \in \operatorname{Crit}_{\mathrm{e}}(f)$ and then $\left[c_{0}\right]=\left\{c_{0}\right\}$, we know that $\bigcup_{c \in\left[c_{0}\right]} K_{n}(c)=$ $K_{n}\left(c_{0}\right)$ and obviously $K_{n}\left(c_{0}\right)$ is nice.

Summarizing Lemmas 5.12, 5.13 and 5.14, we have proved Proposition 5.3.

\section{Appendix A. An applichtion of Theorem 1.3}

Cui and Peng proved the following result in [4] (see Theorem 1.1 in [4]).

Theorem A.1. Let $U$ be a multiply-connected fixed (super)attracting Fatou component of a rational map $f$. Then there exist a rational map $g$ and a completely invariant Fatou component $V$ of $g$, such that:

(1) $(f, U)$ and $(g, V)$ are holomorphically conjugate, i.e., there is a conformal map mapping from $U$ onto $V$ and conjugating $f$ to $g$,

(2) each Julia component of $g$ consisting of more than one point is a quasi-circle which bounds an eventually superattracting Fatou component of $g$ containing at most one postcritical point of $g$.

Moreover, $g$ is unique up to a holomorphic conjugation.

We say that $(g, V)$ is a holomorphic model for $(f, U)$.

The original proof of the uniqueness of the model $(g, V)$ consists of two parts, Proposition 1.3, restated below, and Proposition 1.4 (showing that the Julia set of the model $g$ carries no invariant line fields). 
Proposition A.2. Suppose that $g$ and $\tilde{g}$ are two rational maps, and that $V$ and $\tilde{V}$ are two completely invariant Fatou components of $g$ and $\tilde{g}$ respectively satisfying the conditions (1) and (2) in Theorem A.1. Then there is a qc map from the Riemann sphere $\overline{\mathbb{C}}$ onto itself conjugating $g$ to $\tilde{g}$ on $\overline{\mathbb{C}}$ and this conjugation is conformal on the Fatou set of $g$.

The original proof of Proposition A.2 in [4] exploited a criterion for the existence of qc extensions due to Kozlovski, Shen and van Strien ([16, Lemma 12.1). The condition 3 of the criterion requires one to find a family of pairwise disjoint topological disks with specific properties. The authors stated that they can choose appropriate puzzle pieces to satisfy condition 3 but they did not mention how to choose them. The second author of this current manuscript pointed out that such choices might be difficult to realize. In this appendix, instead of using the above criterion, we will apply Theorem 1.3 to prove directly Proposition A.2 and thus avoid the difficulty.

Proof of Proposition A.2. First starting from $(g, V)$ and $(\tilde{g}, \tilde{V})$, we construct two proper box mappings $(g: \mathbf{U} \rightarrow \mathbf{V})$ and $(\tilde{g}: \tilde{\mathbf{U}} \rightarrow \tilde{\mathbf{V}})$.

We may assume that the fixed points of $g$ and $\tilde{g}$ in $V$ and $\tilde{V}$ are the point at infinity. By Koenig's Linearization Theorem and Böttcher's Theorem ([22]), we can take a disk $D_{0}=\{|z|>r\} \subset V$ such that

(1) $D_{0} \subset \subset g^{-1}\left(D_{0}\right)$,

(2) $\partial D_{0} \cap\left(\bigcup_{n \geq 1} \bigcup_{c \in \operatorname{Crit}(g)}\left\{g^{n}(c)\right\}\right)=\emptyset$, where $\operatorname{Crit}(g)$ denotes the critical point set of $g$. Let $D_{n}$ be the connected component of $g^{-n}\left(D_{0}\right)$ containing $D_{0}$ for each $n \geq 1$. Then $D_{n} \subset \subset D_{n+1}$ and $V=\bigcup_{n=0}^{\infty} D_{n}$. There is an integer $N_{0}$ satisfying that for any $n \geq 0, g^{-n}\left(D_{N_{0}}\right)$ has only one component, the set $\operatorname{Crit}(g)$ is contained in $g^{-n}\left(D_{N_{0}}\right)$ and every component of $\overline{\mathbb{C}} \backslash \bar{D}_{N_{0}}$ contains at most one component of $\overline{\mathbb{C}} \backslash V$ having critical points. Set $\mathbf{V}:=\overline{\mathbb{C}} \backslash \bar{D}_{N_{0}}$ and $\mathbf{U}:=g^{-1}(\mathbf{V})$. Clearly, $\mathbf{K}_{g}=\overline{\mathbb{C}} \backslash V$. One can check that $(g: \mathbf{U} \rightarrow \mathbf{V})$ is indeed a proper box mapping, and use the above method to build a proper box mapping from a rational map with a completely invariant (super)attracting Fatou component.

By Theorem A.1 (1), there is a conformal map $H: V \rightarrow \tilde{V}$ with $\tilde{g} \circ H=H \circ g$ on $V$. Set $\tilde{\mathbf{V}}:=\overline{\mathbb{C}} \backslash H\left(\bar{D}_{N_{0}}\right)$ and $\tilde{\mathbf{U}}:=\tilde{g}^{-1}(\tilde{\mathbf{V}})$. Then $\mathbf{K}_{\tilde{g}}=\overline{\mathbb{C}} \backslash \tilde{V}$ and $(\tilde{g}: \tilde{\mathbf{U}} \rightarrow \tilde{\mathbf{V}})$ is a proper box mapping.

Since $H: V \rightarrow \tilde{V}$ is a qc conjugacy from $g$ to $\tilde{g}$ and $\mathbf{V}=\overline{\mathbb{C}} \backslash \bar{D}_{N_{0}}, \tilde{\mathbf{V}}=$ $\overline{\mathbb{C}} \backslash H\left(\bar{D}_{N_{0}}\right)$, we know that $H: \mathbf{V} \backslash \mathbf{K}_{g} \rightarrow \tilde{\mathbf{V}} \backslash \mathbf{K}_{\tilde{g}}$ is a qc conjugacy off $\mathbf{K}_{g}$. Let $E$ be a periodic critical component of $\mathbf{K}_{g}$ which is mapped to a periodic critical component of $\mathbf{K}_{g}$ under some forward iterate of $g$. According to Theorem A.1 (2), the component $E$ is a quasi-circle which bounds an eventually superattracting Fatou component containing a critical point $c$. In the proof of Proposition 4.4 in [4, a qc map $h_{E}$ is constructed. That map is defined on a puzzle piece $P_{n_{E}}(c)$ containing $E$. From the definition of that map, one can easily check that $\left.h_{E}\right|_{\partial P_{n_{E}}+n}(c)=H$ for all $n \geq 0$. Set $N_{c}:=n_{E}$ and let $M_{c}$ be the maximal dilatation of the map $h_{E}$. Then by Theorem 1.3, $H$ extends to a qc conjugacy off int $\mathbf{K}_{g}$. Notice that every component of $\operatorname{int} \mathbf{K}_{g}$ is a bounded eventually superattracting Fatou component. So $H$ extends to a conformal map in every component of $\operatorname{int} \mathbf{K}_{g}$ which is again a conjugacy (refer to the proof of Claim 4.1 in [4]). Thus $H$ extends to a qc conjugacy on $\mathbf{V}$, that is, $H$ extends to a qc conjugacy on $\overline{\mathbb{C}}$ which is conformal on the Fatou set of $g$. 


\section{ACKNOWLEDGEMENTS}

The authors would like to thank Guizhen Cui, Jeremy Kahn and Weixiao Shen for inspiring discussions. The authors would also like to express our gratitude to the anonymous referees for helpful comments.

\section{REFERENCES}

[1] Lars V. Ahlfors, Lectures on quasiconformal mappings, 2nd ed., University Lecture Series, vol. 38, American Mathematical Society, Providence, RI, 2006. With supplemental chapters by C. J. Earle, I. Kra, M. Shishikura and J. H. Hubbard. MR2241787 (2009d:30001)

[2] Artur Avila, Jeremy Kahn, Mikhail Lyubich, and Weixiao Shen, Combinatorial rigidity for unicritical polynomials, Ann. of Math. (2) 170 (2009), no. 2, 783-797, DOI 10.4007/annals.2009.170.783. MR2552107 (2011d:37078)

[3] Bodil Branner and John H. Hubbard, The iteration of cubic polynomials. II. Patterns and parapatterns, Acta Math. 169 (1992), no. 3-4, 229-325, DOI 10.1007/BF02392761. MR:1194004 (94d:30044)

[4] GuiZhen Cui and WenJuan Peng, On the structure of Fatou domains, Sci. China Ser. A 51 (2008), no. 7, 1167-1186, DOI 10.1007/s11425-008-0056-5. MR2417486 (2009e:30050)

[5] Guizhen Cui and Lei Tan, A characterization of hyperbolic rational maps, Invent. Math. 183 (2011), no. 3, 451-516, DOI 10.1007/s00222-010-0281-8. MR.2772086 (2012c:37088)

[6] A. Douady and J. H. Hubbard, Étude dynamique des polynômes complexes I, II., Publ. Math. dOrsay, 1984-1985.

[7] Adrien Douady and John Hamal Hubbard, On the dynamics of polynomial-like mappings, Ann. Sci. École Norm. Sup. (4) 18 (1985), no. 2, 287-343. MR816367 (87f:58083)

[8] Adrien Douady and John H. Hubbard, A proof of Thurston's topological characterization of rational functions, Acta Math. 171 (1993), no. 2, 263-297, DOI 10.1007/BF02392534. MR.1251582 (94j:58143)

[9] J. H. Hubbard, Local connectivity of Julia sets and bifurcation loci: three theorems of J.-C. Yoccoz, Topological methods in modern mathematics (Stony Brook, NY, 1991), Publish or Perish, Houston, TX, 1993, pp. 467-511. MR1215974 (94c:58172)

[10] H. Inou and M. Shishikura, The renormalization for parabolic fixed points and their perturbation, preprint.

[11] J. Kahn, A priori bounds for some infinitely renormalizable quadratics: I. Bounded primitive combinatorics, arXiv:math/0609045.

[12] Jeremy Kahn and Mikhail Lyubich, A priori bounds for some infinitely renormalizable quadratics. II. Decorations (English, with English and French summaries), Ann. Sci. Éc. Norm. Supér. (4) 41 (2008), no. 1, 57-84. MR2423310 (2009k:37106)

[13] Jeremy Kahn and Mikhail Lyubich, A priori bounds for some infinitely renormalizable quadratics. III. Molecules, Complex dynamics, A K Peters, Wellesley, MA, 2009, pp. 229-254, DOI 10.1201/b10617-7. MR2508259 (2010f:37078)

[14] Jeremy Kahn and Mikahil Lyubich, The quasi-additivity law in conformal geometry, Ann. of Math. (2) 169 (2009), no. 2, 561-593, DOI 10.4007/annals.2009.169.561. MR2480612 (2010a:37091)

[15] Jeremy Kahn and Mikhail Lyubich, Local connectivity of Julia sets for unicritical polynomials, Ann. of Math. (2) 170 (2009), no. 1, 413-426, DOI 10.4007/annals.2009.170.413. MR2521120(2010h:37094)

[16] O. Kozlovski, W. Shen, and S. van Strien, Rigidity for real polynomials, Ann. of Math. (2) 165 (2007), no. 3, 749-841, DOI 10.4007/annals.2007.165.749. MR2335796 (2008m:37063)

[17] Oleg Kozlovski and Sebastian van Strien, Local connectivity and quasi-conformal rigidity of non-renormalizable polynomials, Proc. Lond. Math. Soc. (3) 99 (2009), no. 2, 275-296, DOI 10.1112/plms/pdn055. MR2533666(2011a:37096)

[18] Genadi Levin, Multipliers of periodic orbits of quadratic polynomials and the parameter plane, Israel J. Math. 170 (2009), 285-315, DOI 10.1007/s11856-009-0030-0. MR2506328 (2011d:37082)

[19] Genadi Levin, Rigidity and non-local connectivity of Julia sets of some quadratic polynomials, Comm. Math. Phys. 304 (2011), no. 2, 295-328, DOI 10.1007/s00220-011-1228-7. MR:2795323(2012c:37089) 
[20] Mikhail Lyubich, Dynamics of quadratic polynomials. I, II, Acta Math. 178 (1997), no. 2, 185-247, 247-297, DOI 10.1007/BF02392694. MR1459261 (98e:58145)

[21] John Milnor, Local connectivity of Julia sets: expository lectures, The Mandelbrot set, theme and variations, London Math. Soc. Lecture Note Ser., vol. 274, Cambridge Univ. Press, Cambridge, 2000, pp. 67-116. MR1765085 (2001b:37073)

[22] John Milnor, Dynamics in one complex variable, 3rd ed., Annals of Mathematics Studies, vol. 160, Princeton University Press, Princeton, NJ, 2006. MR2193309 (2006g:37070)

[23] Wenjuan Peng, Weiyuan Qiu, Pascale Roesch, Lei Tan, and Yongcheng Yin, A tableau approach of the KSS nest, Conform. Geom. Dyn. 14 (2010), 35-67, DOI 10.1090/S1088-417310-00201-8. MR2600535 (2011d:37079)

[24] WenJuan Peng and Lei Tan, Combinatorial rigidity of unicritical maps, Sci. China Math. 53 (2010), no. 3, 831-848, DOI 10.1007/s11425-010-0022-x. MR2608337 (2011i:37060)

[25] Feliks Przytycki, Iterations of rational functions: which hyperbolic components contain polynomials?, Fund. Math. 149 (1996), no. 2, 95-118. MR1376666 (97e:58199)

[26] WeiYuan Qiu and YongCheng Yin, Proof of the Branner-Hubbard conjecture on Cantor Julia sets, Sci. China Ser. A 52 (2009), no. 1, 45-65, DOI 10.1007/s11425-008-0178-9. MR2471515 (2009j:37074)

[27] P. Roesch, On local connectivity for the Julia set of rational maps: Newton's famous example, Ann. of Math. (2) 168 (2008), no. 1, 127-174, DOI 10.4007/annals.2008.168.127. MR2415400 (2009c:37043)

[28] M. Shishikura, Yoccoz puzzle, $\tau$-functions and their applications, Unpublished, 1998.

[29] Lei Tan and Yongcheng Yin, The unicritical Branner-Hubbard conjecture, Complex dynamics, A K Peters, Wellesley, MA, 2009, pp. 215-227. MR2508258 (2010f:37077)

[30] William P. Thurston, On the geometry and dynamics of iterated rational maps, Complex dynamics, A K Peters, Wellesley, MA, 2009, pp. 3-137, DOI 10.1201/b10617-3. Edited by Dierk Schleicher and Nikita Selinger and with an appendix by Schleicher. MR2508255 (2010m:37076)

[31] J. -C. Yoccoz, On the local connectivity of the Mandelbrot sets, Unpublished, 1990.

[32] Yongcheng Yin and Yu Zhai, No invariant line fields on Cantor Julia sets, Forum Math. 22 (2010), no. 1, 75-94, DOI 10.1515/FORUM.2010.004. MR.2604364(2011d:37077)

[33] Yu Zhai, Rigidity for rational maps with Cantor Julia sets, Sci. China Ser. A 51 (2008), no. 1, 79-92, DOI 10.1007/s11425-007-0124-2. MR2390757(2009i:37110)

Institute of Mathematics, Academy of Mathematics and Systems Science, Chinese Academy of Sciences, 100190 Beijing, People's Republic of China

E-mail address: wenjpeng@amss.ac.cn

Faculté des Sciences, LAREMA, Université D'Angers, 2, Boulevard Lavoisier, 49045 Angers Cedex 01, France

E-mail address: Lei.Tan@univ-angers.fr 